\title{
Light-Activated Ion Pumps and Channels for Temporally Precise Optical Control of Activity in Genetically Targeted Neurons ${ }^{1}$
}

\section{Brian Y. Chow, Xue Han, Jacob G. Bernstein, Patrick E. Monahan, and Edward S. Boyden}

\begin{abstract}
The ability to turn on and off specific cell types and neural pathways in the brain, in a temporally precise fashion, has begun to enable the ability to test the sufficiency and necessity of particular neural activity patterns, and particular neural circuits, in the generation of normal and abnormal neural computations and behaviors by the brain. Over the last 5 years, a number of naturally occurring light-activated ion pumps and light-activated ion channels have been shown, upon genetic expression in specific neuron classes, to enable the voltage (and internal ionic composition) of those neurons to be controlled by light in a temporally precise fashion, without the need for chemical cofactors. In this chapter, we review three major classes of such genetically encoded "optogenetic" microbial opsins-light-gated ion channels such as channelrhodopsins, light-driven chloride pumps such as halorhodopsins, and light-driven proton pumps such as archaerhodopsins - that are in widespread use for mediating optical activation and silencing of neurons in species from Caenorbabditis elegans to nonhuman primates. We discuss the properties of these moleculesincluding their membrane expression, conductances, photocycle properties, ion selectivity, and action spectra-as well as genetic strategies for delivering these genes to neurons in different species, and hardware for performing light delivery in a diversity of settings. In the future, these molecules not only will continue to enable cutting-edge science but may also support a new generation of optical prosthetics for treating brain disorders.
\end{abstract}

Key words: Channelrhodopsin, Optogenetics, Photosensitive proteins, Retinal, Halorhodopsin, Archaerhodopsin, Light-sensitive cation channel, Light-sensitive chloride pump, Light-sensitive proton pump, Photocontrol of behavior

\section{Introduction}

The ability to turn on and off specific cell types and neural pathways in the brain, in a temporally precise fashion, has begun to enable the ability to test the sufficiency and necessity of particular neural activity patterns, and particular neural circuits, in the generation of normal

\footnotetext{
${ }^{1}$ This chapter is an updated version of reference (141).
} 
and abnormal neural computations and behaviors by the brain. Most electrophysiological and imaging experiments in neuroscience are correlative-comparing a neural signal observed in the brain to a behavior or pathology. In contrast, the power to manipulate specific cells and circuits is opening up the ability to understand their causal roles in brain functions. Over the last 5 years, a number of naturally occurring light-activated ion pumps and light-activated ion channels have been shown, upon genetic expression in specific neuron classes, to enable the voltage (and internal ionic composition) of those neurons to be controlled by light in a temporally precise fashion. These molecules are microbial (type I) opsins, seven-transmembrane proteins naturally found in archaea, algae, fungi, and other species, and which possess light-driven electrogenic activity or contain light-gated ion pores. These molecules, when heterologously expressed in neurons or other cells, translocate ions across cell membranes in response to pulses of light of irradiances that are easily achievable with common laboratory microscopes, LEDs, and lasers. These molecules have begun to find widespread use in neuroscience due to their ease of handling and use (each is a single gene, under $1 \mathrm{~kb}$ long, encoding for a monolithic protein), their lack of need for chemical supplementation in many species (they utilize the naturally occurring chromophore all-trans retinal, which appears to occur at sufficient quantities in the mammalian nervous system), and their high speed of operation (they can respond within tens of microseconds to milliseconds, upon delivery of light, and shut off rapidly upon cessation of light, as needed for neuroscience experiments).

Three major classes of such "optogenetic" microbial opsins have been described to date. The first class, channelrhodopsins, is exemplified by the light-gated inwardly rectifying nonspecific cation channel channelrhodopsin-2 (ChR2) from the green algae Chlamydomonas reinhardtii (1), which, when expressed in neurons, can mediate sizeable currents up to $1,000 \mathrm{pA}$ in response to millisecond-timescale pulses of blue light $(2-5)$, thus enabling reliable spike trains to be elicited in ChR2-expressing neurons by blue light pulse trains (Fig. 1b). Several additional channelrhodopsins useful to biologists and bioengineers have been discovered or engineered, with faster or slower kinetics, red-shifted activation, and cell-region specific targeting, and explored in detail below (6-10). The channelrhodopsins have been used to activate neurons in neural circuits in animals from worms to monkeys, and have proven powerful and easy to use. The second class of microbial opsins utilized for biological control to date, halorhodopsins, is exemplified by the light-driven inwardly directed chloride pump halorhodopsin, from the archaeal species Natronomas pharaonis (Halo/NpHR/pHR; (11)), which, when expressed in neurons, can mediate modest inhibitory currents on the order of 40-100 pA in response to yellow light illumination $(12,13)$, enabling moderate silencing of neural activity (Fig. 1c). Halorhodopsins have some intrinsic kinetic limitations, 
ai

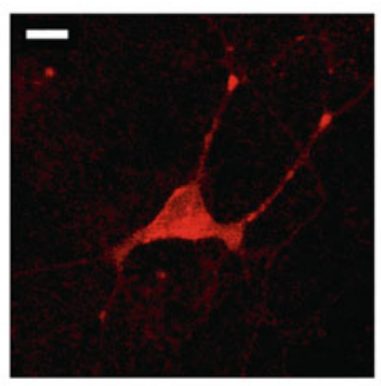

aii

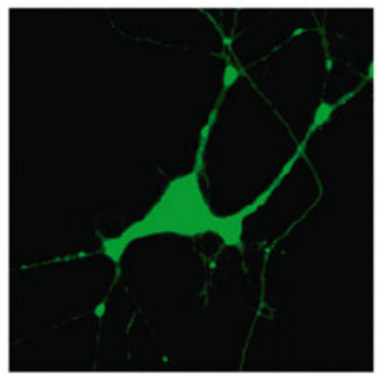

b

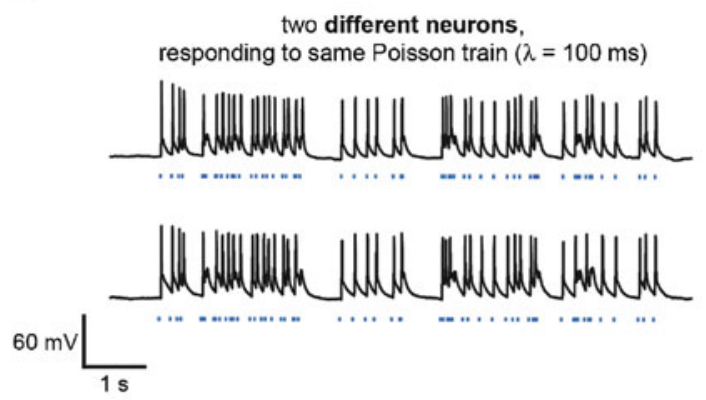

c
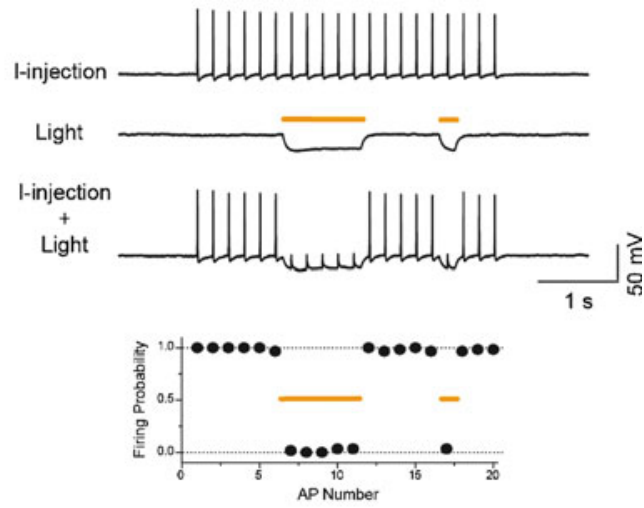

e
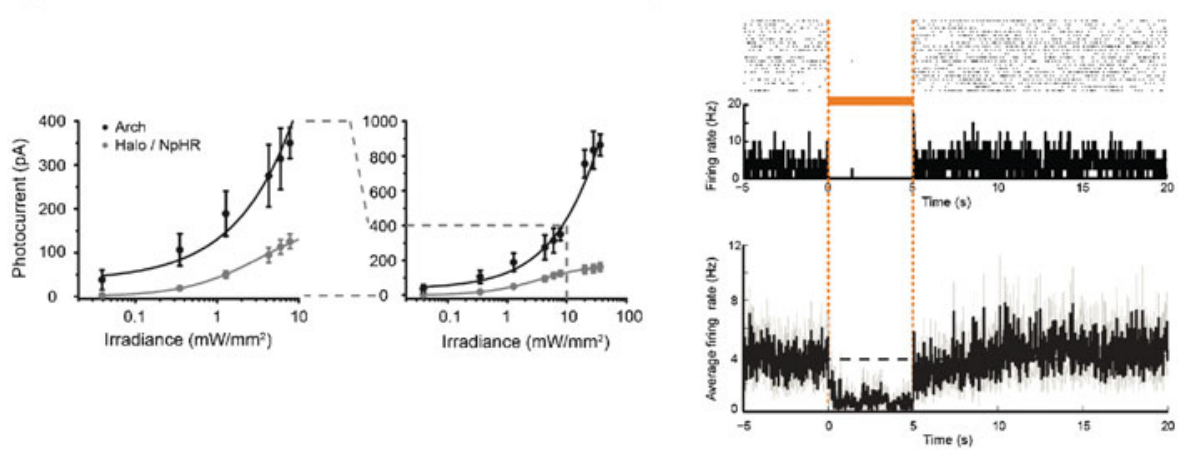

Fig. 1. Three classes of microbial opsins that enable optical neural activation and silencing tools. (a) Neuron expressing hChr2-mCherry (ai; bar, $20 \mu \mathrm{m}$ ) and Halo-GFP (aii). (b) Poisson trains of spikes elicited by pulses of blue light (blue dashes), in two different neurons. (c) Light-driven spike blockade, demonstrated (Top) for a representative hippocampal neuron, and (Bottom) for a population of neurons $(N=7)$. I-injection, neuronal firing induced by pulsed somatic current injection (300 pA, $4 \mathrm{~ms})$. Light, hyperpolarization induced by periods of yellow light (yellow dashes). I-injection + Light, yellow light drives Halo to block neuron spiking, leaving spikes elicited during periods of darkness intact. Panels a-c adapted from references Boyden et al. (3) and Han and Boyden, (12). (d) Photocurrents of Arch vs. Halo measured as a function of $575 \pm 25 \mathrm{~nm}$ light irradiance (or effective light irradiance), in patch-clamped cultured neurons $(N=4-16$ neurons for each point), for low (i) and high (ii) light powers. The line is a single Hill fit to the data. (e) Top, Neural activity in a representative neuron before, during, and after $5 \mathrm{~s}$ of yellow light illumination, shown as a spike raster plot (top), and as a histogram of instantaneous firing rate averaged across trials (bottom; bin size, $20 \mathrm{~ms}$ ). Bottom, population average of instantaneous firing rate before, during and after yellow light illumination (black line, mean; gray lines, mean $\pm \mathrm{SE}$; $n=13$ units). Panels $\mathbf{d}-\mathbf{e}$ adapted from reference Chow et al. (15). 
with some photocycles taking tens of minutes to complete (e.g., Fig. $4 \mathrm{a}, \mathrm{b} ;(12,14))$, and halorhodopsins also require improved trafficking for expression at high levels (15-17). A third class of microbial opsin, the bacteriorhodopsins, is exemplified by the lightdriven outward proton pump archaerhodopsin-3 (Arch/aR-3), from the archaeal species Halorubrum sodomense. Arch can mediate strong inhibitory currents of up to $900 \mathrm{pA}$ in vitro (Fig. 1d), and in vivo is capable of mediating near-100\% silencing of neurons in the awake behaving mouse or monkey brain in response to yellow-green light (Fig. 1e, $(15,18))$. Protons are extremely effective as a charge carrier for mediating neural silencing, and proton pumps have greatly improved kinetics with respect to halorhodopsins (Fig. 4c, d), as well as a fast photocycle, and efficient trafficking to membranes. Furthermore, outward proton pumps, perhaps surprisingly, do not alter $\mathrm{pH}$ to a greater extent than do other opsins (such as ChR2) or than does normal neural activity. The broad and ecologically diverse class of outward proton pumps, which includes blue-green light drivable outward proton pumps such as the Leptosphaeria maculans opsin Mac (Fig. 5), enables, alongside the yellow-red drivable Halo, multicolor silencing of independent populations of neurons (15). Also, because the neural silencers Halo and Arch are activated by yellow or yellow-green light, and the neural depolarizer ChR2 is driven by blue light, expression of both a silencer and a depolarizer in the same cell (either by using two viruses, or by using the 2A linker to combine two opsins into a single open reading frame $(12,19))$ enables bidirectional control of neural activity in a set of cells, useful for testing necessity and sufficiency of a given set of neurons in the same animal, or disruption of neural synchrony and coordination through "informational lesioning" (19).

In the sections below we describe the properties of these three opsin classes, as well as genetic (e.g., viral, transgenic) and hardware (e.g., lasers, LEDs) infrastructures for using these opsins to parse out the function of neural circuits in a wide variety of animal nervous systems. A theme of this field is that extremely rapid progress and adoption of these technologies has been driven by technology development curves in other fields such as gene therapy and optical imaging. Our hope is to convey a snapshot of this rapidly moving field as of 2011 , summarizing the first half-decade of its existence, to teach both neuroengineers hoping to innovate by inventing new tools, as well as neuroscientists hoping to utilize these tools to answer new scientific questions. We will first survey the general properties of these opsins (Sect. 2), then go into the channelrhodopsins (Sect. 3), followed by the neural silencing pumps (halorhodopsins and bacteriorhodopsins, Sect. 4), the molecular strategies for delivering these genes to cells for appropriate expression in vitro and in vivo (Sect. 5), and the hardware for illumination of these opsin-expressing neurons in vitro and in vivo (Sect. 6). 


\section{Properties of "Optogenetic" Microbial (Type I) Opsins}

The three classes of molecule described to date are from organisms such as unicellular algae, fungi, and archaea, whose native environments and membrane lipid composition are very different from those of mammalian neurons. Thus, the performance of these molecular tools in neurons can be difficult to predict based solely upon their properties in other species, and must be assessed empirically for assurance of efficacy and safety. Nevertheless, there are several molecular properties that contribute to efficacious, temporally precise optical control of neurons, which can be explored in a unified and logical fashion:

- Initial protein expression levels. The efficiency of ribosomal translation of a molecule is largely affected by codon optimization. It is recommended that genes be used that are codonoptimized for the target species.

- Membrane insertion properties, protein folding, and interactions with local environment. Increased membrane localization will result in more functional molecules and thus increased photocurrents. This property may also be inversely associated with the potential property of toxicity, since poorly trafficked or folded molecules may aggregate in the cytosol and endoplasmic reticulum. On the other hand, if a molecule has adverse intrinsic side effects, enhanced trafficking may exaggerate them. Furthermore, any given channel or pump will best operate under defined conditions (e.g., chloride conductance, $\mathrm{pH}$, lipid environment, etc.), which may not exist in a given target cell type.

- Innate conductance and permeability. Channels translocate more ions per photocycle than pumps, since they open up a pore in the membrane. On the other hand, pumps can move ions against concentration gradients, unlike channels. Each opsin furthermore passes a precise set of ions in a specific cellular context, and not others.

- Photocycle kinetics. Both light-driven channels and pumps are described by a photocycle, the list of states that a molecule goes through after light exposure, including ion-translocating or ion pore-forming steps. The faster the photocycle, the more temporally precise the molecular function might be, and for a pump, the more ions will be translocated. (For a channel, a faster photocycle may result in the channel entering the ion pore-forming state more often, but may also reduce the time spent in the open state.) If a molecule enters an inactive 
photocycle state for an enduring period of time, it may be effectively nonfunctional.

- Photosensitivity. Molecules may require different amounts of light to begin moving through their photocycle, based on the chromophore absorption efficiency. Furthermore, from a enduser standpoint, effective photosensitivity will appear to be a function of the overall photocycle; for example, a pump that has a slow photocycle may appear to be light insensitive (because incident photons may have no effect on the molecule during the photocycle), whereas a channel that inactivates extremely slowly may appear to be light sensitive (because each photon will result in large charge transfers).

- Action spectrum. Different molecules are driven by different colors of light. Multiple cell types can be orthogonally addressed with different colors of light, if they express opsins whose action spectra minimally overlap.

- Ion selectivity. Unlike traditional electrodes, microbial opsins can generate ion specific currents, since they will pass specific ions such as chloride $\left(\mathrm{Cl}^{-}\right)$or calcium $\left(\mathrm{Ca}^{+2}\right)$. This opens up novel kinds of experimental capability, such as the ability to test the sufficiency of a given ion, in a given location, for a given biological function.

We will, in the following sections, frame current knowledge about cell-type specific optical control of neurons, in the context of decades of research in structure-function relationships of microbial (type I, or archaeal) opsins. In many ways, these molecules are similar in tertiary structure to mammalian (type II) rhodopsins (20), the pigments that confer photosensitivity to the rods and cones of the human retina. Both types are composed of seven transmembrane (7-TM) $\alpha$-helices, linked by six loop segments, and their photosensitivity is enabled by a retinal bound to a specific lysine residue near the C-terminus, forming a Schiff base that undergoes a trans-cis or cis-trans isomerization upon illumination, that then induces conformational changes in the protein. However, they are evolutionarily unrelated, and their differences have important implications for their use in perturbing neuronal activity. Mammalian rhodopsins (21) are very sensitive photon detectors, optimized for sensitivity rather than speed. They utilize 11-cis retinal as the primary chromophore, which isomerizes to all-trans retinal upon absorbing a photon. The resultant structural change activates an associated G-protein, transducin, which then initiates a cascade of secondary messengers. The all-trans retinal dissociates from the opsin, is converted back to its 11-cis form, and then reassociates with the apoprotein to reconstitute a functional molecule-a process that typically takes hundreds of milliseconds, too slow to enable fast control of neurons in the central nervous system. 
On the other hand, a microbial opsin utilizes all-trans retinal as its chromophore, which isomerizes to 13-cis retinal upon absorbing a photon. The chromophore does not undergo a quasi-irreversible dissociation event, but rather thermally relaxes to its active all-trans form in the dark (although this process can be facilitated by light). The trans-cis isomerization sets off several coupled structural rearrangements within the molecule that accommodate the passive conduction or active pumping of ions (22-24). Ultimately, this means that at the expense of light sensitivity, archaeal opsins can deflect the membrane potential of a cell on the scale hundreds of microseconds to a few milliseconds. However, it should be noted that genetically targetable, optical neural silencing has also been demonstrated using mammalian G-protein coupled receptors, which can couple to potassium channels (4), and genetically targetable optical neural activation has been demonstrated using melanopsins and invertebrate-style rhodopsins, at the price of temporal precision $(25,26)$.

As an exemplar of a well-characterized microbial opsin reagent, with crystal structure and photocycle both well-described, Fig. 2 shows the crystal structure of the light-driven chloride-pump halorhodopsin (Fig. 2a; (27)), as well as schematized structural rearrangements that are hypothesized to occur as the molecule pumps a chloride ion across the membrane and into the cytoplasm (Fig. 2b; (24)). While it is convenient to consider the molecular tools discussed here as toggle switches for turning neurons on and off, it is critical for use of these opsins to realize that the translocation of ions by microbial opsins is not as simple as a two-state toggle switch. The structural rearrangements constitute an active advancement through a complex photocycle with various intermediate states beyond the initial phototransition (Fig. 2c). There exists a very rich literature on type I microbial opsins from an evolutionary and protein structure-function perspective. The canonical molecules include the proton-pumping bacteriorhodopsin (BR), the chloride-pumping halorhodopsin (HR/sHR/HsHR) from Halobacterium salinarum (halobium), and the halorhodopsin from $N$. pharaonis (Halo/ $\mathrm{NpHR} / \mathrm{pHR}$ ). These were some of the first membrane proteins crystallized, and a myriad of structure-function studies have been performed on these molecules $(11,14,20$, 22-24, 27-45). These studies are not reviewed here, but it is important to point out their existence because much of what we know with respect to the photocycle and structure of opsins comes from these studies and from sequence homology of novel opsins to these canonical molecules. As we show later, for example, a deep understanding of the literature has enabled some researchers to derive powerful new variants of channelrhodopsin $(5,6,8,10)$, even though no crystal structure exists for this molecule. 
a

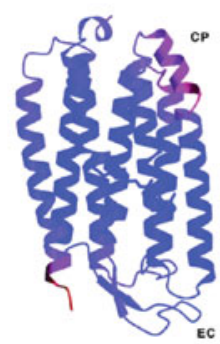

c

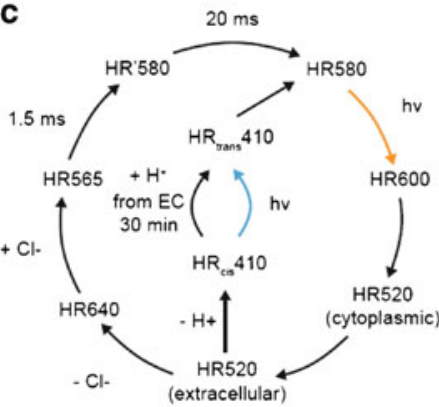

b
HR580
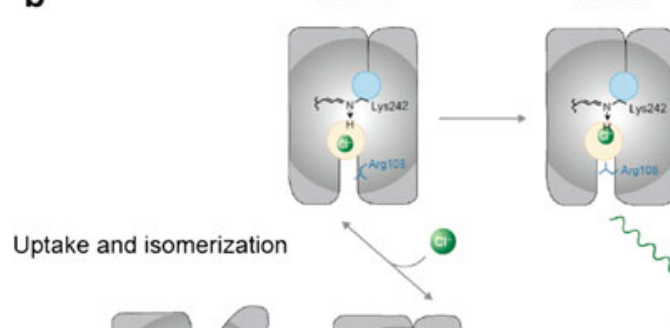

पn

Isomerization

HR640

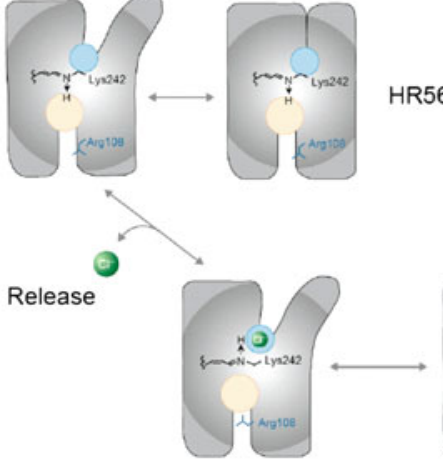

HR565

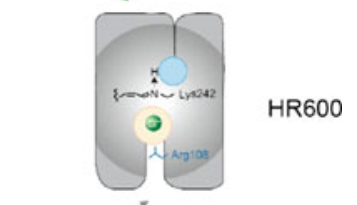

Switch

HR520

Fig. 2. Structure and function of halorhodopsin. (a) Crystal structure of halorhodopsin, which is composed of 7-transmembrane $\alpha$-helices (7-TM) and a retinal chromophore that forms a Schiff base to a lysine near the C-terminus (from Kolbe et al. (27)). (b) Schematic of the halorhodopsin structural rearrangements and their relation to pumping activity at various points in the photocycle. Image modified from Essen (24). (c) The halorhodopsin photocycle at high-power continuous illumination on the timescale of a typical photocycle (i.e., conditions used for neural silencing, $>$ few milliseconds). The HR410 intermediate is the origin of the long-lived inactivation in neural silencing (e.g., Bamberg et al. (14); Han and Boyden (12)).

\section{Optical Neural \\ Stimulation: Channel-rhodopsins}

Channelrhodopsins are the primary photoreceptors in the eyespot of the unicellular algae that are responsible for phototactic and photophobic responses (46-48). Their name is derived from the fact that despite the sensory function, the 7-TM segment is in itself a light-activated ion channel. While the channel pore and properties remain poorly understood, it has recently been realized that channelrhodopsins are likely proton pumps like many other microbial opsins, but with a leaky step in the photocycle during which the opsin lets positive charge into cells (49). In C. reinhardtii, two separate channelrhodopsins were originally identified (48), one with fast kinetics but poor light sensitivity, channelrhodopsin-1 (ChRl) (50), and another with slower kinetics but improved sensitivity, channelrhodopsin-2 (ChR2) (1). Two more channelrhodopsins from Volvox carteri (VChRl, VChR2) have also been identified $(7,51)$, and as we discuss later, many more ChRs are expected to 


\subsection{Conductance, Permeability, and Context}

exist. ChRl-style channelrhodopsins have red-shifted action spectra $\left(\right.$ peak $\left.\lambda_{\mathrm{ChRl}}=500 \mathrm{~nm}, \lambda_{\mathrm{VChRl}}=535 \mathrm{~nm}\right)$ relative to ChR2 (peak $\lambda_{\mathrm{ChR} 2}=470 \mathrm{~nm}$ ), and thus in principle ChRl-style and ChR2-style opsins could be used together to drive separate sets of neurons with two different colors of light, if suitably spectrally separated opsin pairs could be found.

Channelrhodopsins (abbreviated as ChRs or chops) are lightactivated, inwardly rectifying cation channels that are, at neutral $\mathrm{pH}$, permeable to physiologically relevant cations such as $\mathrm{H}^{+}, \mathrm{Na}^{+}$, $\mathrm{K}^{+}$, and $\mathrm{Ca}^{2+}$, with permeabilties (relative to sodium) of $1 \times 10^{6}, 1$, 0.5 , and 0.1 respectively $(1,6,46,50)$. It is of particular note that the proton conductance $\left(\mathrm{G}_{\mathrm{H}_{+}}\right)$is $10^{6}$-fold larger than the sodium conductance $\left(\mathrm{G}_{\mathrm{Na}+}\right)$, and thus near physiological $\mathrm{pH}$, perhaps half the photocurrent is carried by protons (46); thus, ChRs may rapidly equilibrate the intracellular $\mathrm{pH}$ with its environment (10). Kinetic selectivity analysis has shown that the mechanism of ion selectivity is likely to be due to differential binding affinity of channelrhodopsin channel residues for different ions, not differential ion transport rates (46).

It was originally believed that $\mathrm{ChRl}$ was a selective proton channel (50); however, it was later discovered that the poor photocurrents at mammalian $\mathrm{pH}$ were likely attributable to poor membrane localization (6), and the apparent lack of sodium currents in the original report were due to the low $\mathrm{pH}$ used to perform experiments in that study; the sodium conductance of ChRl lessens at low $\mathrm{pH}(6,46)$, unlike that of ChR2 (6). This highlights what is a recurrent theme throughout this chapter, that effective conductance in a heterologous system is determined not only by the innate kinetic and transport properties of the molecule but also by its trafficking and performance in the environment of the heterologous system.

The single ion channel conductance of ChR2 has been estimated at $50 \mathrm{fS}(1)$, which corresponds to approximately $3 \times 10^{4}$ ions per second, or 300 ions per photocycle event, assuming a $10 \mathrm{~ms}$ turnover. This is considerably less than a typical voltage dependent sodium channel that may have a conductance on the order of $\sim 10 \mathrm{pS}$. It has been estimated from electrophysiological data that $10^{5}-10^{6}$ membrane embedded ChR2 molecules are required to cause reliable spiking in cultured rodent hippocampal neurons (52), with saturation blue light densities of several milliwatts per square millimeter both in vitro (10) and in vivo (53).

Figure 3a shows a typical photocurrent trace from a voltageclamped neuron expressing ChR2 (top), and the spiking pattern that would result in current-clamp mode (bottom). There is a large transient peak with an opening time constant near $1 \mathrm{~ms}(1,6,10)$, although photocurrent onset can be measured at $<200 \mu \mathrm{s}(1,3$, 
a
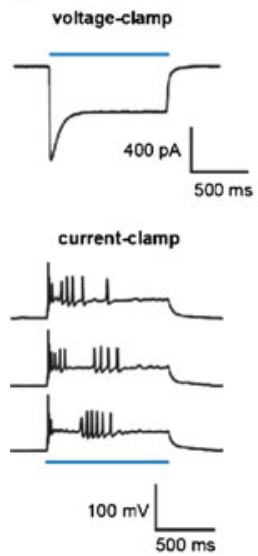

b

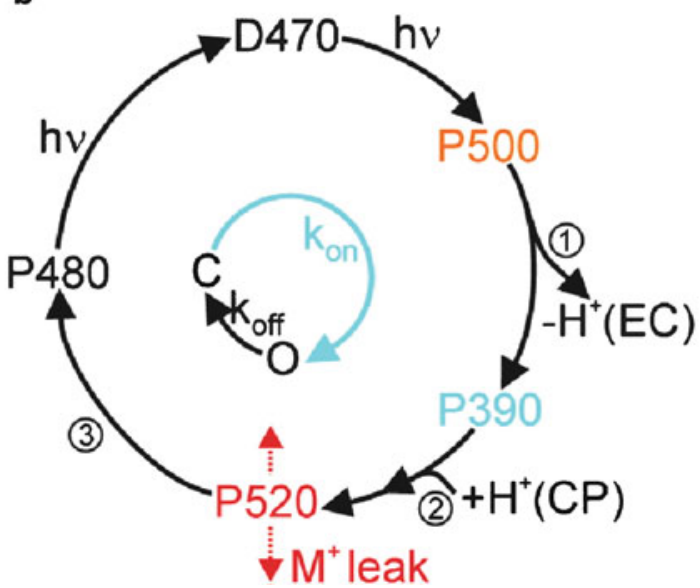

C

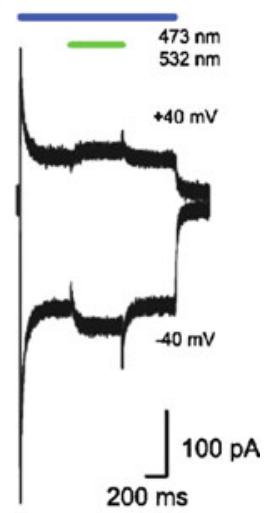

Fig. 3. Channelrhodopsin kinetic and photocycle properties, and impact on neural activity. (a) ChR2 currents elicited in a voltage-clamped hippocampal neuron expressing ChR2 and illuminated by blue light (top), and ChR2-driven spikes elicited in a current-clamped hippocampal neuron (three repetitions of the same blue light pulse in the same neuron) (bottom), under $1 \mathrm{~s}$ of blue light illumination. Adapted from Boyden et al. (3). (b) The photocycle of ChR2 determined by a combination of spectroscopy, site-directed mutagenesis, and electrophysiology, adapted from Feldbauer et al. (49). The inner circle summarizes the effective appearance of the photocycle, an approximation to the outer photocycle. (c) The interplay between photocycle, wavelength, and electrophysiological activity. ChR2 is excited with blue light for a brief period, and then a green light is turned on. The photocurrent initially diminishes because the channel is forced to close, but then increases because the green light also pumps the molecule into its most highly efficient state. Image modified from Bamann et al. (54).

$54)$; this transient peak quickly decays to a stationary component that is typically $<20-50 \%$ of the initial peak photocurrent $(1,3,6,10)$. Upon removing the light, ChR2 closes with a time constant of 10-20 ms $(1,6,10)$. The transient photocurrent peak is highly dependent on the illumination intensity $(51,55)$ and history $(1,3$, $10)$; the history dependence results from a desensitization of the transient component that takes $\sim 5$ s to recover from in the dark (3). The stationary component on the other hand, is less photosensitive and effectively history-independent (55). ChR2 absorbs maximally at $460 \mathrm{~nm}(1,10)$, and the action spectra of both temporal components are nearly identical in ChR2.

The large and fast-onset peak enables ChR2-expressing neurons to spike with exquisite temporal precision on the millisecond timescale (Fig. 1b), the timescale of an action potential. However, the large inactivation (or alternatively, the small stationary component) and its slow recovery in the dark, as well as the slow closing rate of $\sim 10-15 \mathrm{~ms}$, ultimately limit the ability to drive reliable spike rates $>25 \mathrm{~Hz}(3,10)$ because (1) the stationary photocurrent may be too small to sufficiently depolarize a neuron to spike threshold, and (2) the channel cannot physically close quickly enough to enable de-inactivation of sodium channels. It should be noted, though, that many neurons, such as pyramidal cells, seldom fire 
action potentials at this rate on the individual neuron level (vs. population synchrony or rhythmogenesis).

ChRl-style channelrhodopsins (VChRl, ChRl) $(7,50)$ on the other hand demonstrate dramatically faster kinetics than ChR2-style channelrhodopsins (VChR2, ChR2). The stationary photocurrents of ChRls are $>70 \%$ of the peak photocurrents, and the channels open and close approximately two- to threefold faster than does ChR2. Therefore, one would expect that given comparable expression, protein folding, membrane localization, and photosensitivity (i.e., factors contributing to effective conductance), ChRls would be capable of driving spike rates with greater fidelity than ChR2s. However, poor membrane expression limits the performance of natural ChRl-style channelrhodopsins $(7,50)$. Chimeras composed of the first five helices of ChRl and last two helices of ChR2 have been constructed $(6,10,56)$, and these new variants exhibit the small inactivation and action spectrum of $\mathrm{ChRl}$, but the overall effective conductance of ChR2. These structure-function studies are discussed in detail later in this chapter. A point mutant of this chimera dubbed "ChIEF" (based on its composition as a [Ch] annelrhodopsin chimera with an [I]190 V substitution with domains swapped between ChRl helix-[E] and ChR2 helix-[F]), developed by Tsien and coworkers (10), appears to be a highly improved tool for stimulating neurons. Its large stationary photocurrent and very fast channel closing, the latter conferred by the I190 V mutation, contribute to far more reliable spiking (up to $100 \mathrm{~Hz}$ ) than ChR2.

Based on the available characterization of the channelrhodopsins from $V$. carteri $(7,51)$, the general characteristics are similar to those of the analogous molecules in C. reinhardtii. VChR2 and ChR2 have nearly identical photocycles and action spectrum (5l). VChRl and ChRl exhibit the similar reduced inactivation, and are both red-shifted from their respective VChR2/ChR2 counterparts. It has been proposed that VChRl could be used for multicolor optical stimulation in conjunction with ChR2, which is blue-shifted by $\sim 70 \mathrm{~nm}$, but further improvements are likely required for reliable spiking because the VChRl photocurrents are unfortunately approximately four- to fivefold smaller (7), and also there is significant spectral overlap between VChRl and ChR2.

3.3. Detailed Models of Kinetics and Wavelength Selectivity
As previously mentioned, it is critical to realize that the translocation of ions by opsins is not as simple as the operation of an on/off switch, but rather these opsins traverse a complex photocycle with various intermediate states beyond the initial opening of the channel. Figure $3 \mathrm{~b}$ shows the photocycle of ChRs based on photophysical studies performed primarily by laser flash spectroscopy, physiology, and site-directed mutagenesis (8, 49, 51, 54, 57). Importantly, the intermediates of the photocycles themselves can also undergo photoreactions, and thus they may be optically driven 
or "short-circuited" (54) between photointermediates at much faster rates (Fig. 3c). The ChR2 photocycle initially begins in its closed dark-adapted state D470 (where the number in the state name corresponds to the peak light absorption, in $\mathrm{nm}$, of the molecule in that state). The channel opens when D470 absorbs a photon, after which the molecule will become a green absorbing photoproduct or P-intermediate, P520, via thermal relaxation from shorter lived photoproducts. This initial cascade of events takes $0.2-1.5 \mathrm{~ms}$, depending on the transmembrane potential. The open ChR2 can be closed by either optically pumping P520 $\rightarrow$ D470 with green light, or by decaying to P480 (via a yet to be determined intermediate), a process that takes $\sim 6 \mathrm{~s}$. The inactivation toward the stationary photocurrent may be due to molecules making the $\mathrm{P} 520 \rightarrow \mathrm{P} 480$ transition, rather than the optically induced P520 $\rightarrow$ D470 transition that would allow the molecule to quickly open again. Assuming that $\mathrm{ChRl}$ and ChR2 photocycles are topologically similar, e.g., the ChR2 D470 and P480 equate to the ChRl peaks at 464 and $505 \mathrm{~nm}$, this interpretation of the transient and stationary photocurrents is consistent with the finding that, for $\mathrm{ChRl}$, the stationary photocurrent is redshifted from the transient photocurrent (6).

The various wavelengths of absorption of rhodopsins and their intermediates throughout the photocycle arises from the different conformations of the chromophore and its local environment, which influences the chromophore charge distribution and the protonation of the retinylidene Schiff base. Figure $3 \mathrm{c}$ demonstrates this complex interplay in an experiment by Bamberg and coworkers (54). After blue light-excited ChR2 has reached its steady state, a green light costimulation is introduced. The photocurrent briefly diminishes because the open channel is forced to close, but the stationary photocurrent quickly improves because many molecules have been pumped back into their highly efficient, peak producing state. Thus, it is possible that slightly red-shifted or broadband illumination of ChR2 may strike a balance between optimally exciting the dark state (transient component) and repriming the dark state (driving the red-shifted intermediate photoproduct). As we discuss, optimal silencing with $N$. pharaonis halorhodopsin is analogously achieved by using both yellow light to hyperpolarize the neuron and blue light to drive the molecule out of its inactive state $(12,14)$.

3.4. Mutants and Variants
As previously mentioned, even though no ChR crystal structure exists at the time of this writing, useful structure-function studies have been performed based largely on sequence homology to $H$. salinarum bacteriorhodopsin. The E90Q mutation (57) has increased sodium selectivity (with respect to $\mathrm{G}_{\mathrm{H}_{+}}$) vs. wild-type ChR2, and the H134R mutant (5) demonstrates increased conductance by approximately twofold. Various mutations to C128 (8) 
corresponding to bacteriorhodopsin T90, drastically slow down the rate of ChR2 closure from the open state, thus effectively creating a bistable open P520 state until illuminated with green light. By lengthening the time that $\mathrm{ChR} 2$ spends open on a per-photon basis, this mutation effectively decreases the amount of light needed to activate the channel, at the expense of temporal precision. In contrast, the E123T mutant, combined with the H134R mutant, speeds channel closure and increases the precision of neural action potential firing at the expense of photocurrent and light sensitivity (58), resulting in a reagent nicknamed ChETA.

Chimeras of $\mathrm{ChRl}$ and $\mathrm{ChR} 2$ have been constructed by several researchers $(6,10,56)$, one of which was that composed of ChRl helices A-E and ChR2 helices F-G (called abcdeFG, ChEF, or $\mathrm{ChRl} / 2_{5 / 2}$ by various investigators). These chimeras displayed the small inactivation of ChRl, but the large photocurrents of ChR2 on account of improved membrane localization and light sensitivity (based on quantitative confocal fluorescence microscopy, (6)). An I190 V substitution to ChEF led to the molecule, "ChIEF," capable of driving more reliable fast spiking due to the much larger stationary current and faster channel closing kinetics after light offset (10). During these studies, it was also discovered that a single point mutation to wild-type ChRl, E87Q, eradicates its $\mathrm{pH}$-dependent spectral shifts, and increases inactivation during illumination (56).

The fact that the poor effective conductance of ChRl can be largely attributed to membrane localization rather than its photophysical properties highlights the importance of considering and improving the trafficking of heterologously expressed molecules. In particular, ChRs are not localized to the outer membrane, but rather the eyespot, in C. reinhardtii, and the membrane composition of the organism is less than $20 \%$ phosphoglyceride (59), a primary lipid type in mammalian neurons. As we later discuss in the context of halorhodopsins, the use of signaling peptides can improve outer membrane localization and reduce aggregation in the cytosol, endoplasmic reticulum, and Golgi apparatus.

Along similar lines of using signal peptides to alter trafficking, the myosin binding domain (MBD) peptide promotes subcellular localization of opsins to neuronal dendrites (9). This subcellular localization strategy may prove to be helpful for enabling driving of electrical activity in specific neural compartments, or for high-resolution connectomic mapping in vivo. Two-photon excitation is a powerful laser excitation technique that enables submicron resolution in 3-D (60) relatively deep into the brain $(\sim 750 \mu \mathrm{m}$, or a significant fraction of the thickness of the mouse cortex), but its ability to induce action potentials in a neuron expressing ChR2 is limited by the interplay between molecule density and the extent of optical depolarization with respect to time $(61,62)$. The probability of inducing an action potential, at low powers 


\subsection{Diversity}

that are not destructive to tissue, is relatively low using a traditional raster scan because the fraction of molecules excited at any point in time is small, and most photons that do hit the membrane are wasted (since the open time of ChR2 is long relative to a femtosecond laser photon delivery rate). Thus, with most conventional twophoton laser scanning methods, the aggregate contributions of the serially excited molecules never sufficiently depolarize the whole neuron to spike threshold. However, Rickgauer and Tank have demonstrated that neurons expressing ChR2 can be reliably excited by two-photon microscopy by optimizing the scan pattern to deliver light optimally to the cell membrane, in a fashion that reaches the maximum surface area while minimizing wastage of photons on already-light-driven channelrhodopsin molecules (62).

Unlike microbial rhodopsins from archaea, significantly less is known about the photoelectrogenic molecules of unicellular algae, the only organisms known to date to have naturally occurring light-activated channels. Photoelectric responses have been measured in several green flagellates, as well as phylogenetically distant cryptophytes (47, 63-65). Interestingly, the two-component phototaxis strategy employed by C. reinhardtii, in which the response is mediated by a fast $(\mathrm{ChRl})$ and slow $(\mathrm{ChR} 2)$ rhodopsin, appears to be general (47), which begs the question whether chimeras of their respective rhodopsins will also result in kinetic improvements and variants with interesting properties. Thus, as more phototaxis-mediating rhodopsins are isolated and sequenced, or as perhaps new depolarizing rhodopsin types are discovered, new molecular tools for controlling neurons will surely emerge.

\section{Optical Neural Silencing: Halorho- dopsins and Bacteriorho- dopsins}

Whereas traditional electrodes can stimulate neurons with temporal precision (albeit without cell type specificity), they are incapable of silencing neurons in order to assess their necessity for given neural computations, behaviors, and pathologies. Therefore, there is a large need for spatio-temporally precise methods for optical inhibition of neurons. Inwardly rectifying chloride pumps and outwardly rectifying proton pumps, halorhodopsins (HRs, hops) and bacteriorhodopsins (BRs, bops), respectively, are electrogenic pumps that when heterologously expressed are capable of sufficiently hyperpolarizing a neuron to silence its activity (Fig. lc-e; $(12,15,66))$. They are thus far known to exist in every kingdom except for animals: archaea $(22,23,67-69)$, bacteria $(70-76)$, fungi $(77,78)$ and algae $(79)$. In addition to their opposite electrophysiological effect, HRs and BRs differ primarily 
from channelrhodopsins in that their physiological functions is chiefly due to their role as pumps as opposed to operating as passive channels, and thus can translocate ions against concentration gradients (but, typically only one ion per photocycle). Much is known about the photocycles and structure-function relationships of HRs and BRs because they have been crystallized $(24,27,28,80,81)$ and heavily characterized via spectroscopy, mutagenesis, and physiology for decades.

This section focuses on two molecules in particular: N. pharaonis halorhodopsin (Halo/ $\mathrm{NpHR}$ ) and H. sodomense bacteriorhodopsin (Arch/AR-3), also known as an archaerhodopsin (that is, a bacteriorhodopsin from the halorubrum genus). Halorhodopsins were shown in 2007 to be capable of mediating modest optical neural hyperpolarizations, and since have been improved in trafficking to boost their currents $(12,13,16)$; bacteriorhodopsins were shown in 2009 to be able to mediate very powerful and kinetically versatile silencing of multiple neural populations with different colors of light (18). We discuss in the following sections "Conductance, permeability, and context" and "Kinetics and wavelength selectivity" of halorhodopsins and bacteriorhodopsins for these two classes separately, followed by a joint discussion of the "Mutants and variants" and genomic "Diversity" in a unified section.

4.1. Halorhodopsins: Conductance, Permeability, and Context
N. pharaonis halorhodopsin (NpHR, Halo) is a highly selective, inwardly rectifying, chloride pump, which can also conduct larger monovalent anions (82). It has a reversal potential of approximately $-400 \mathrm{mV}(82)$, and its chloride-dependence of pumping activity (full- and half-saturating chloride concentrations: $\left[\mathrm{Cl}^{-}\right]_{\text {saturation }}=20$ $\left.\mathrm{mM},\left[\mathrm{Cl}^{-}\right]_{1 / 2}=2.5 \mathrm{mM}\right)(83)$ is appropriate for operation in mammalian cells, in contrast to $H$. salinarum halorhodopsin, which is not capable of effective operation in mammalian neurons (15), presumably because of its large chloride dependency: $\left[\mathrm{Cl}^{-}\right]_{\text {saturation }}$ $=5 \mathrm{M}$ and $\left[\mathrm{Cl}^{-}\right]_{1 / 2}=200 \mathrm{mM}(83,84)$. In the absence of any signal peptide sequences to improve trafficking and membrane localization, Halo has been reported to generate $40-100$ pA of hyperpolarizing current $(12,18,66)$, with the differences in measured photocurrents between studies largely attributable to the power and wavelength of excitation used. This photocurrent is approximately 10-25-fold less than typical peak depolarizing currents generated by ChR2, highlighting one potential disadvantage inherent to a pump that translocates one ion per photocycle (e.g., Halo) vs. a channel that conducts 300 ions per photocycle (e.g., ChR2). To mediate these currents, there are an estimated ten million membrane-embedded Halo molecules per neuron (as assessed in hippocampal neuron culture). Because the expression levels are so high, Halo is known to form puncta or intracellular blebs, aggregating in the endoplasmic reticulum (ER) and Golgi apparatus $(16,17)$. These issues are somewhat addressed by attaching trafficking enhancement sequences to 
4.2. Halorhodopsins: Kinetics and Wavelength Selectivity the molecule, e.g., sequences from the KiR2.1 protein (eNpHR, eNpHR3.0), which increases the effective conductance several fold by increasing membrane expression $(16,85)$.

Recently, we have discovered that the crux-halorhodopsin (HR from the baloarcula genus) from Haloarcula marismortui, canonically known as cHR-5 $(69,86)$, produces similar photocurrents to Halo with more uniform expression; even when highly overexpressed under high copy number transfection conditions, no puncta or intracellular blebbing is observed (15). This molecule may better express than Halo in vivo, but it is unknown at this moment whether it will ultimately be more efficacious at altering mammalian behavior, given their statistically insignificant difference in photocurrent. However, the prolactin (Prl) ER-location sequence in conjunction with a signal sequence from a MHC class I antigen triples the Halo photocurrent $(15,18)$; we are now trying out multiple trafficking sequences in combination to see if they boost current further. However, it is important to note that if halorhodopsins have other side effects that are due to the protein's intrinsic properties-as an example, one paper quantitates the significantly altered neuronal capacitance that results from expressing halorhodopsin in neurons in vivo (87) - then boosting expression may only make such side effects worse.

$N$. pharaonis halorhodopsin is capable of silencing weakly firing neurons on the millisecond timescale with its $\sim 100 \mathrm{pA}$-scale currents, with rapid onset and offset (12), but during long periods of illumination, all halorhodopsins that we have tested so far and that have current (from N. pharaonis, H. sodomense, Haloarcula vallismortis, H. marismortui, and Salinibacter ruber) inactivate by approximately $30 \%$ every $15 \mathrm{~s}$ of illumination at $1-10 \mathrm{~mW} / \mathrm{mm}^{2}$ yellow $(593 \mathrm{~nm}$ ) light (Fig. 4a, b; $(12,18))$. This slow inactivation stands in contrast to ChR2, which responds to light with a large transient peak that decays within seconds, followed by a stable stationary photocurrent. For all of the halorhodopsins named above, recovery in the dark from light-induced inactivation is slow, with a time constant of tens of minutes, as has been described for some halorhodopsins earlier $(12,14,39)$ (Fig. 4a, b). This longlasting inactivation property may hinder the use of halorhodopsins for silencing for prolonged periods, e.g., during repeated behavioral trials. Importantly, for all halorhodopsins investigated, the inactive photoproduct can be driven back into its active pumping state with a short (e.g., subsecond duration) pulse of blue or UV light $(12,14)$; thus, optimal use of Halo for neural silencing requires both yellow and blue light to be delivered to the same set of neurons, which is possible (88) but can complicate optics setups.

The Halo photocycle is shown in schematic form in Fig. 2c. The time constants listed are the limiting ones, with all other transitions $<100 \mu \mathrm{s}$. It should be noted that the names for 
a

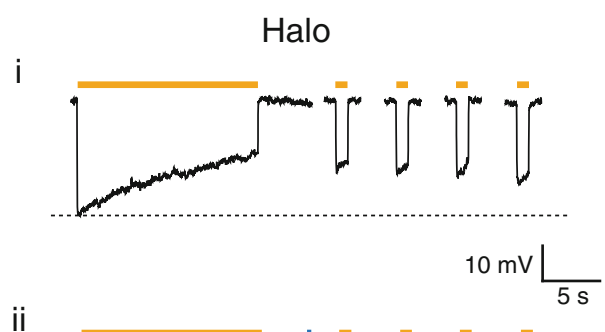

ii

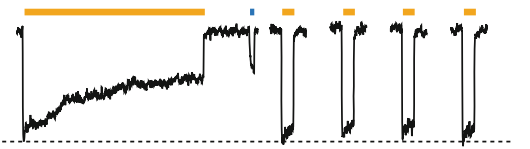

b

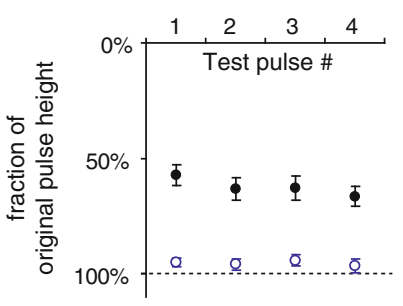

C

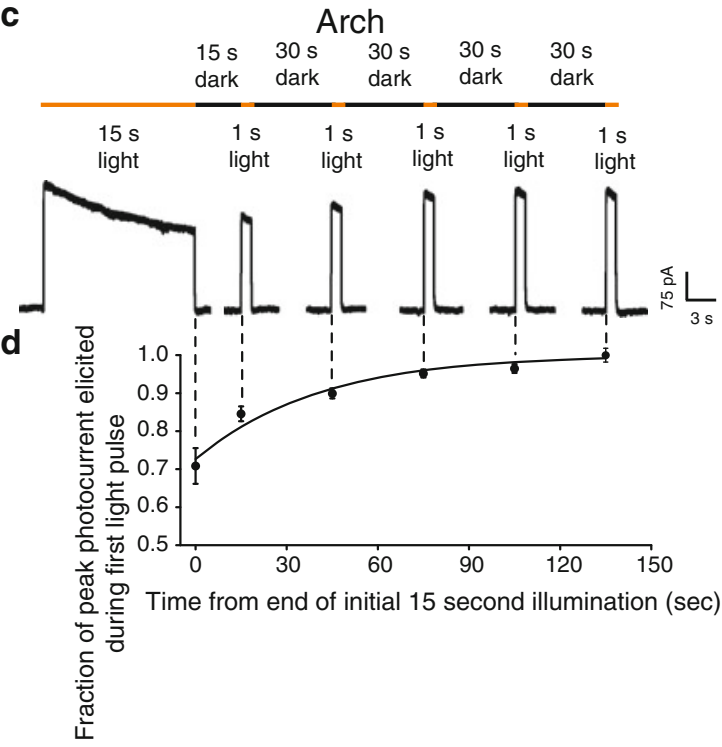

Fig. 4. Kinetic comparisons between halorhodopsins and archaerhodopsins. (ai) Time course of Halo-mediated hyperpolarizations in a representative current-clamped hippocampal neuron during $15 \mathrm{~s}$ of continuous yellow light, followed by four 1-s test pulses of yellow light (one every $30 \mathrm{~s}$, starting $10 \mathrm{~s}$ after the end of the first 15-s period of yellow light). (aii) Time course of Halo-mediated hyperpolarization for the same cell exhibited in (ai), but when Halo function is facilitated by a 400-ms pulse of blue light in between the 15-s period of yellow light and the first 1-s test pulse. (b) Population data for blue-light facilitation of Halo recovery ( $N=8$ neurons). Plotted are the hyperpolarizations elicited by the four 1-s test pulses of yellow light, normalized to the peak hyperpolarization induced by the original 15-s yellow light pulse. Dots represent mean \pm S.E.M. Black dots represent experiments when no blue light pulse was delivered (as in Fig. 5ai.). Open blue dots represent experiments when $400 \mathrm{~ms}$ of blue light was delivered to facilitate recovery (as in Fig. 5aii.). (c) Raw current trace of a neuron lentivirally infected with Arch, illuminated by a $15 \mathrm{~s}$ light pulse ( $575 \pm 25 \mathrm{~nm}$, irradiance $7.8 \mathrm{~mW} / \mathrm{mm}^{2}$ ), followed by $1 \mathrm{~s}$ test pulses delivered starting $15,45,75,105$, and $135 \mathrm{~s}$ after the end of the $15 \mathrm{~s}$ light pulse. (d) Population data of averaged Arch photocurrents $(N=11$ neurons) sampled at the times indicated by the vertical dotted lines that extend into Fig. 4c.

canonical spectroscopic states of H. salinarum halorhodopsin-the $\mathrm{K}, \mathrm{L}, \mathrm{N}$, and $\mathrm{O}$ photointermediates-have not been used here because the order of the $\mathrm{N}$ - and $\mathrm{O}$-states in $\mathrm{N}$. pharaonis halorhodopsin is still somewhat debated $(42,89,90)$. In the dominant photocycle, Halo absorbs a photon and then within tens of microseconds, quickly releases a chloride ion into the cytoplasm during the HR520 $\rightarrow$ H640 transition, via short-lived intermediates that "switch" the chloride location within the molecule from the extracellular loading domain to the cytoplasmic release domain. The molecule then reisomerizes from the HR640 state and takes up a chloride ion from the extracellular side, a process that takes $\sim 1.5 \mathrm{~ms}$; it then forms the HR' state, which finally relaxes to the active ground state with a time constant of $\sim 20 \mathrm{~ms}$. However, 
4.3. Bacteriorhodopsins: Conductance, Permeability, and Context halorhodopsins can enter an alternate photocycle (middle trajectory within Fig. 2c), most notably under prolonged or bright illumination, as might occur during in vivo neural silencing. The 13- cis retinalydene Schiff base becomes deprotonated (releasing a proton into the cytoplasm and thus introducing a small depolarizing proton current) and forms a long-lived intermediate HR410 $(14,39)$. In the dark, the halorhodopsin will remain in this inactive state for a duration on the order of $30 \mathrm{~min}$ (39). This formation of HR410 is the origin of the long inactivation observed in neurons expressing halorhodopsin and the ability to recover the active state using the short blue light pulse $(12,14)$. In contrast, as we discuss in detail in the next section, archaerhodopsins (bacteriorhodopsins from the Halorubrum genus) spontaneously recover in the dark under physiological conditions.

Arch, canonically known as archaerhodopsin-3 (AR-3) from $H$. sodomense, is a yellow-green-light sensitive outwardly rectifying proton pump with nearly an order of magnitude increase in hyperpolarizing current over any characterized natural halorhodopsin $(15,18)$, attaining neuronal currents up to $900 \mathrm{pA}$ in vitro in response to light powers easily achievable in vitro or in vivo. The efficacy of these proton pumps is surprising, given that protons occur, in mammalian tissue, at a millionfold-lower concentration than the ions carried by the optical control molecules described above. This high efficacy may be due to the fast photocycle of Arch ( see also $(91,92)$ ), but it may also be due to the ability of high-pKa residues in proton pumps to mediate proton uptake $(91,93)$.

Arch is a highly efficacious tool in vivo, with cortical neurons in the awake behaving mouse undergoing a median of $97.1 \%$ reductions in firing rate for periods of seconds to minutes (Fig. le) (18), and safely expresses for months in both mice and monkeys when virally delivered in vivo. Due to the larger currents, Arch enables very large (e.g., order of magnitude-scale) increases in addressable volume of tissue silenceable, over earlier reagents. We thoroughly investigated the safety of Arch function. To date, blebbing issues that have affected the usage of halorhodopsins have not been observed in vitro or in vivo for Arch, but membrane trafficking sequences may still prove effective at boosting expression beyond the natural state (the Prl sequence, which greatly magnifies Halo current, slightly increases Arch current in neurons). Furthermore, we have not observed changes in cell membrane capacitance or other passive neural properties, as has been reported with halorhodopsin expression (see above). From a end-user standpoint, illumination of Arch neurons was safe: spike rates measured in vivo were not significantly different before vs. after periods of optical neural silencing. Biophysically, $\mathrm{pH}$ changes in neurons expressing Arch and undergoing illumination were minimal, plateauing rapidly at alkalinizations of $\sim 0.1-0.15 \mathrm{pH}$ units; the fast stabilization of $\mathrm{pH}_{\mathrm{i}}$ 
may reflect a self-limiting influence that rapidly limits proton concentration swings, and may contribute to the safe operation of Arch in neurons, as observed in mice and monkeys. Indeed, the changes in $\mathrm{pH}$ observed in cells expressing Arch and being illuminated are comparable in magnitude to those observed during illumination of ChR2-expressing cells (10) (due to the proton currents carried by ChR2 $(1,46))$ and are also within the magnitudes of changes observed during normal neural activity (94-97). We have observed that other archaerhodopsins from other Halorubrum strains are also particularly powerful molecular reagents (work in progress). In contrast, the canonical $H$. salinarum bacteriorhodopsin, well known to poorly function in Escherichia coli, successfully produced modest photocurrents in mammalian neurons, which highlights the importance of not assuming all molecules will express and traffic the same in different organisms. (In contrast, E. coli does not support any detectable expression of ChR2, which expresses well in neurons; target species influences in modulation of opsin function should not be underestimated).

4.4. Bacteriorhodopsins: Kinetics and Wavelength Selectivity
Unlike all of the halorhodopsins we have screened to date (including not only the natural halorhodopsins described above, but also products of halorhodopsin site-directed mutagenesis aimed at improving kinetics), which after illumination remained inactivated for tens of minutes, Arch spontaneously recovers function in seconds in the dark (Fig. 4c, d), more like the light-gated cation channel channelrhodopsin-2 (ChR2) than like halorhodopsins. This feature is particularly useful for in vivo behavior work because it dramatically simplifies the necessary optical hardware; the need to use only one wavelength of light also increases the available bandwidth for multicolor silencing in multiple cell-types. This spontaneous recovery has also been observed by us with other archaerhodopsins, and thus may be a general feature of archaerhodopsins as a whole (work in progress).

Arch is maximally excited with green-yellow light $(\lambda=561 \mathrm{~nm})$, a fairly common peak wavelength for proton pumps. Thus, it is backward compatible with halorhodopsin-driving equipment. Proton pumps naturally exist that are activated by many colors of light, in contrast to chloride pumps, which are primarily driven by yelloworange light (even with significant mutagenesis of retinal-flanking residues, (15)). The light-driven proton pump from L. maculans, here abbreviated Mac, has an action spectrum strongly blue-shifted relative to that of the light-driven chloride pump Halo (Fig. 5a). We found that Mac-expressing neurons could undergo 4.1-fold larger hyperpolarizations with blue light than with red light, and Haloexpressing neurons could undergo 3.3-fold larger hyperpolarizations with red light than with blue light, when illuminated with appropriate powers and filters (Fig. 5b). Accordingly, we could demonstrate selective silencing of spike firing in Mac-expressing 
a

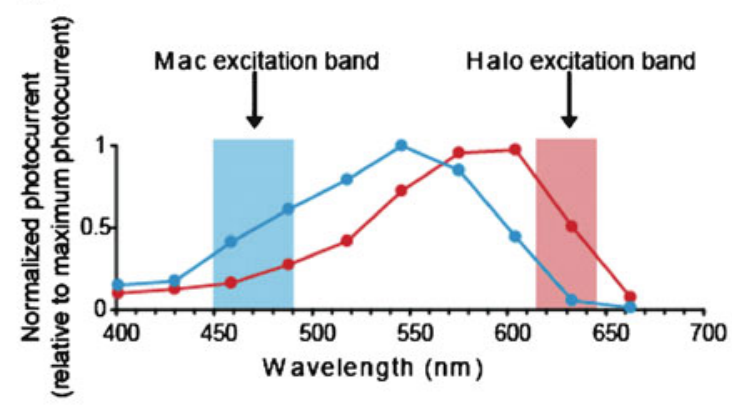

b

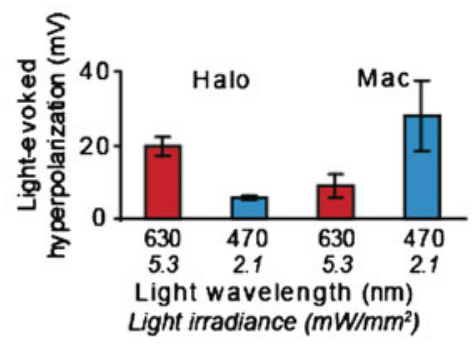

C

i Halo-expressing neuron
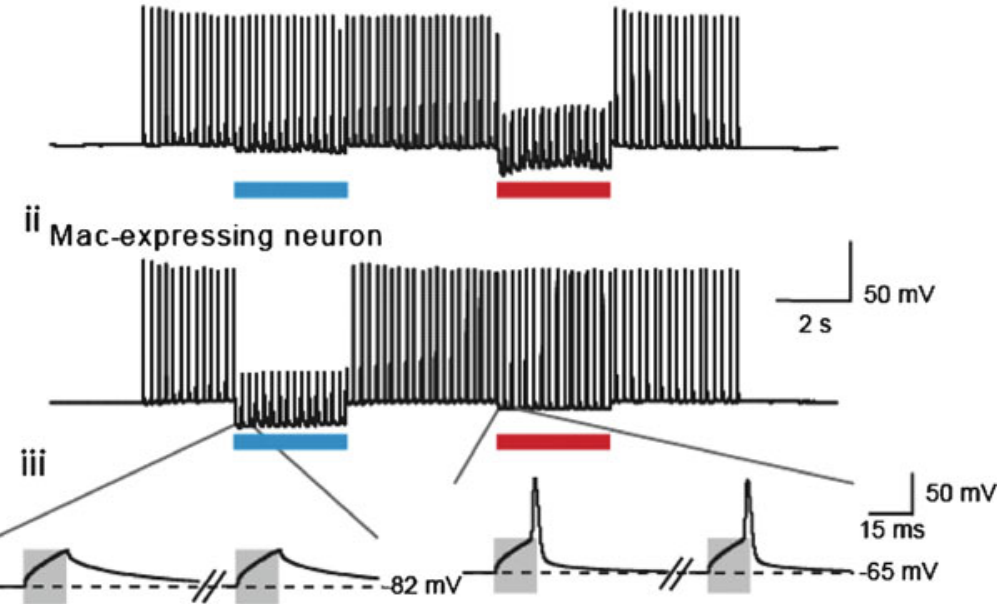

Fig. 5. Multicolor silencing of two neural populations, enabled by blue- and red-light drivable ion pumps of different classes. (a) Action spectra of Mac vs. Halo; rectangles indicate filter bandwidths used for multicolor silencing in vitro. Blue light power is via a $470 \pm 20 \mathrm{~nm}$ filter at $5.3 \mathrm{~mW} / \mathrm{mm}^{2}$, and red light power is via a $630 \pm 15 \mathrm{~nm}$ filter at $2.1 \mathrm{~mW} / \mathrm{mm}^{2}$. (b) Membrane hyperpolarizations elicited by blue vs. red light, in cells expressing Halo or Mac $(N=5$ Mac-expressing neurons, $N=6$ Halo-expressing neurons). (c) Action potentials evoked by current injection into patch clamped cultured neurons transfected with Halo (ci) were selectively silenced by the red light but not by the blue light, and vice versa in neurons expressing Mac (cii). Gray boxes in the inset (ciii) indicate periods of patch clamp current injection.

neurons in response to blue light, and selective silencing of spike firing in Halo-expressing neurons in response to red light (Fig. 5c). Thus, the spectral diversity of proton pumps points the way toward independent multicolor silencing of separate neural populations. 


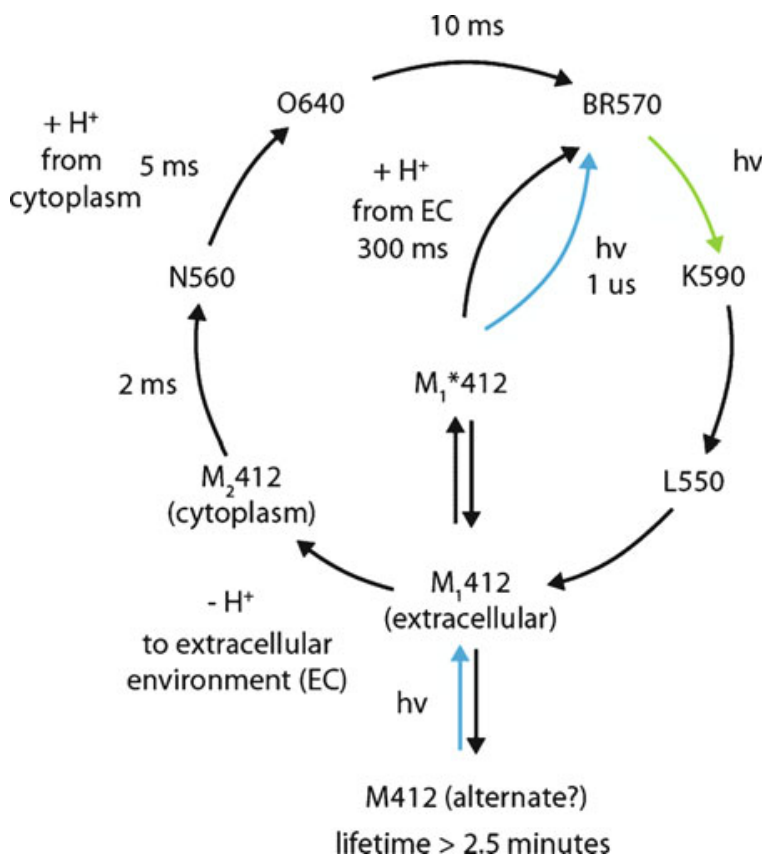

Fig. 6. The photocycle of the H. salinarum bacteriorhodopsin. As in Figs. $2 \mathrm{c}$ and $3 \mathrm{~b}$, the photocycle has been simplified to reflect the dominant photocycle at large continuous illumination on the timescale of a typical photocycle (i.e., conditions used for neural silencing, $>$ few milliseconds). The M412 alternate intermediate is the origin of long-lived inactivation with bacteriorhodopsin. In contrast, Arch spontaneously quickly recovers from this state in the dark.

This result opens up novel kinds of experiment, in which, for example, two neuron classes, or two sets of neural projections from a single site, can be independently silenced during a behavioral task.

Figure 6 shows the photocycle of the canonical H. salinarum bacteriorhodopsin, as representative of the photocycle of the class of proton pumps (the photocycles of archaerhodopsins are not as well characterized, and may well be different) $(22,93)$. The data that has led to the synthesis of the modern model of the bacteriorhodopsin photocycle provide many of the insights that have led to solid investigations into this field as a whole. As in Figs. $2 \mathrm{c}$ and $3 \mathrm{~b}$, it has been simplified to represent the dominant photocycle expected at large and continuous illumination on the timescale of a typical photocycle (e.g., many milliseconds, as would be used for neural silencing). Upon absorbing a photon, bacteriorhodopsin forms its $\mathrm{L} 550$ intermediate $(\mathrm{L}=\mathrm{lumi})$ within microseconds, after quickly transferring a proton from the retinylidene Schiff base (at the Lys-216 position) to the Asp- 85 proton acceptor. This transfer triggers the proton releasing group (PRG), containing Glu-204 and Glu-194, to release its own proton $(98,99)$, during the $\mathrm{L} \rightarrow \mathrm{M}$ transition. After a "switching" step during which the Schiff base reorients itself on the cytoplasmic side, it is then 
4.5. Halorhodopsins and Bacteriorhodopsins: Mutants and Variants

4.6. Diversity reprotonated during the $\mathrm{M} \rightarrow \mathrm{N}$ transition via the Asp-96 donor residue, which in turn picks up a proton from the cytoplasm in the next transition $(\mathrm{N} \rightarrow \mathrm{O})$. The chromophore reisomerizes to the alltransstate during this transition as well. Finally, the bacteriorhodopsin relaxes back to its ground state as the proton release group is reloaded from the Asp-85 residue. Like halorhodopsin, bacteriorhodopsin can become trapped in a long-lasting light-unresponsive state (Fig. 6, "M412 alternate") that requires blue light to reenter the normal photocycle; this could partly explain the lower currents observed with BR when compared to Arch.

For decades, researchers have been making mutants of bacteriorhodopsins and halorhodopsins for structure-function studies reviewed in many earlier publications (e.g., $(22,24,93)$ ). However, point mutations that improve these molecules as neural silencing tools have yet to be reported. Given that the conductance of a pump is limited by the fact that they move only one ion per photocycle, it would be highly desirable to mutate halorhodopsin into a channel, or channelrhodopsin into an anion channel. Tuning the action spectrum of both bacteriorhodopsins and halorhodopsins may be achieved by mutating the retinal-flanking residues $(75,76,100$, 101). Mutations that alter ion selectivity, such as the most wellknown example of this is the bacteriorhodopsin D85T mutation that converts it into a chloride pump (102), could allow ion-specific currents to be mimicked. $\mathrm{A} \mathrm{Ca}^{2+}$ selective pump, for example, would have powerful impact on enabling powerful studies of plasticity, synaptic transmission, and cellular signaling. Given that crystal structures for many bacteriorhodopsins and halorhodopsins exist, we anticipate that functionally oriented and applied site-directed mutagenesis will be a highly active field of research in neuroengineering. The fact that archaerhodopsins on the whole represent a class of molecules that express particularly well in mammalian cells possibly indicates properties of their lipid-interacting residues, or perhaps "signal sequence"-like activity by their loop regions. The known crystal structures of these molecules $(80,81)$ may provide key insights into the trafficking of heterologously expressed molecules and their membrane insertion.

Light-activated proton and chloride pumps are known to exist in far more organisms than do light-gated cation channels, and proton pumps are particularly prevalent, as all opsins described to date likely have at least some proton pumping capability $(22,23$, $67-79,103,104)$. Even though many proton pumps maximally absorb blue-green to green wavelengths (which opens up, as shown in Fig. 5, the possibility, alongside yellow-light driven chloride pumps, for multiple-color silencing of distinct neural populations), light-activated hyperpolarizing currents carried by protons have been observed across the whole visible spectrum, from deep blue 
(via a sensitizer) (105) to far red (106) (>650 nm), although the red-light sensitive current likely originates from a receptor that triggers an $\mathrm{H}^{+}$-ATPase, as opposed to direct light-mediated ion translocation.

The discovery or creation of a purely genetically encoded lightactivated inhibitory channel would be highly desirable. In addition to seeking natural molecules and site-directed mutants, linking a non-light-gated ion-channel to a type I archaeal rhodopsin may be another promising approach to creating such a molecular tool (107). In this way, a light-activated shunt could be created, which would more closely mimic natural mechanisms of neural inhibition in the brain.

\section{Molecular Targeting of Microbial Opsins to Different Cell Types}

The number of papers using optical neural control in species ranging from C. elegans to mouse to nonhuman primate is increasing exponentially each year, and so we do not attempt to review the literature comprehensively. In each species, opsins have been used to test the necessity and sufficiency of neurons, cell types, muscles, neural pathways, brain regions, and other entities in behaviors, pathologies, and neural computations. Opsins have proven valuable in exploring neural dynamics in multiple mammalian brain structures as well. We focus on highlighting principles that govern how to best use these opsins in various settings, from a molecular biology standpoint (Sect. 5) and from a physical-optical standpoint (Sect. 6). From a molecular biology standpoint, these opsins can be delivered to neurons in almost any conventional way that genes are delivered into cells or into organisms. Transgenic mice have been made with ChR2, for example (108), mice and monkeys have been injected with lentiviruses, adeno-associated viruses (AAV), and other viruses encoding for light-gated proteins (2, 52, 109-111), and rodents and chicks have been electroporated in utero with plasmids encoding for light-gated proteins $(4,53,112)$. In each case, different parameters of the technique can be selected so as to enable specific cell types, pathways, or regions to be selectively labeled or to selectively express the opsin. Transgenic C. elegans have been made expressing ChR2 using conventional methods (5), as have transgenic Drosophila (113) and zebrafish (114). For these latter species, supplementation with all-trans-retinal may be beneficial, whereas mammalian brains seem to operate microbial opsins without need for supplementation. (It is possible that in the future, genetically engineering retinal-lacking organisms, such as invertebrates, to produce retinal within their nervous systems may be of use, e.g., by expressing within them enzymes that can produce retinal from vitamin precursors (115)). 
For mammalian nervous systems, a large variety of possible strategies exist for conveying opsin genes into specific cell types. For example, transgenic mice can be made through BAC transgenic, knock-in, or other methods, but such strategies are not common for other species yet. For viral delivery, cell type specific promoters can be inserted upstream of the opsin to target various excitatory, inhibitory, and modulatory neurons (e.g., (19, 116-119)); the size of the promoter is limited by the virus type (AAV viruses hold typically $4-4.5 \mathrm{~kb}$ total, whereas lentiviruses hold typically $8-10 \mathrm{~kb}$ maximum). The surface or coat proteins that a virus bears can also modulate which cell types will take up the virus; for example, lentiviruses may favor excitatory neurons of the cortex, whereas certain AAV serotypes may favor inhibitory cells (120). Lentiviruses can be pseudotyped-fabricated with a coat protein of desired targeting capacity, e.g., with rabies glycoprotein that leads to lentiviruses that travel retrogradely (121)-whereas AAVs can be engineered with biotinylation sites that enable, upon streptavidin conjugation, targeting of potentially arbitrary substrates (122). Retroviruses, which preferentially label dividing cells, have been used to deliver ChR2 to newborn neurons of the dentate gyrus of the hippocampus (123). Other viruses such as rabies virus and pseudorabies virus can be used, with unique tracing capabilities including the ability to go retrogradely across multiple synapses $(124,125)$. Viral particles typically have to be injected directly into the brain, often through stereotactic targeting to a specific brain area, since the potent blood-brain barrier typically precludes systemic delivery of large viral particles (although see (126)). We have recently described a parallel injector array that can deliver viruses into complex three-dimensional configurations (127). Finally, in utero electroporation of ChR2-GFP into embryonic mice at embryonic day $\sim 15.5$ has been found to selectively label pyramidal cells in layers $2 / 3(53,112)$.

Of potential interest is the possibility of a new generation of neural prosthetics, which can accomplish the synthetic neurobiology mission of repairing the nervous system by enabling optical input of information to sculpt neural dynamics and overcome pathology. To deliver these genes in a safe, efficacious, and enduring way, viruses such as AAV may be valuable; AAV has been used in over 600 human patients in gene therapy clinical trials without a single serious adverse event (128) and has been successfully used with opsin delivery. The ability to optically control specific targets within the brain may enable more potent and sideeffect free therapies than possible with existing electrical and magnetic neuromodulation therapies, or with drugs which often are nonspecific and have side effects. Already several groups have prototyped blindness therapies that may enable new approaches to a 


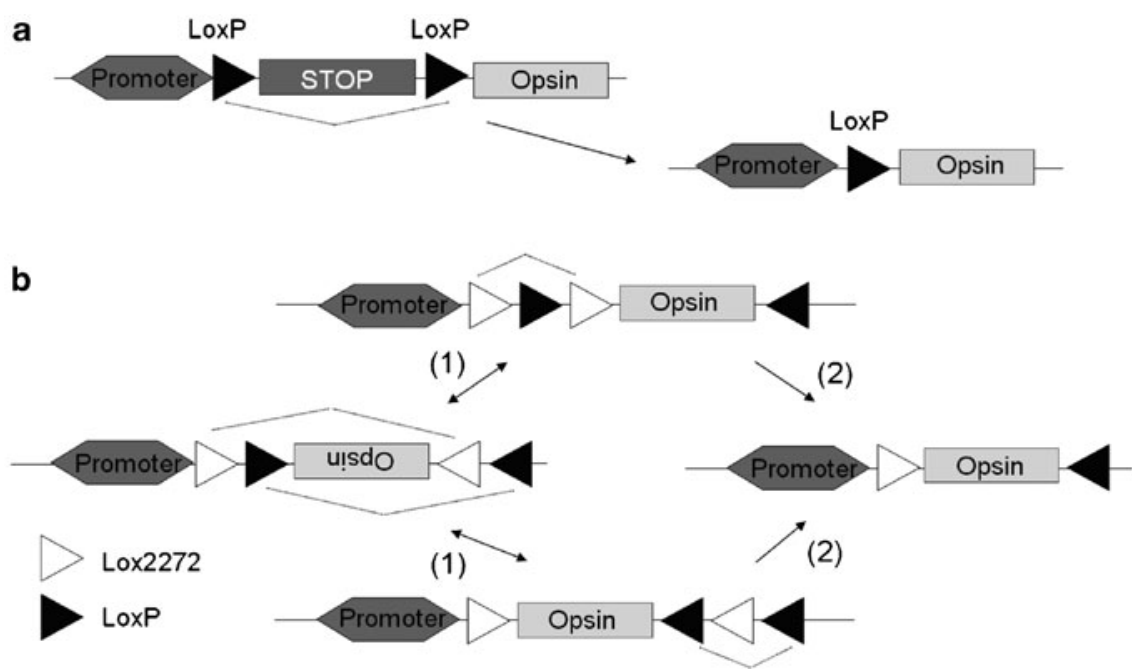

Fig. 7. Transgenic mouse expressing Cre within specific cells, coupled to lox-containing AAV viruses, enable cell-type specific opsin expression. (a) A pair of loxP recombination sequences meditate the removal of the transcriptional and translational stop cassette containing multiple polyadenylation signals (STOP), in the presence of Cre (provided in transgenic mice within specific cell types), to initiate gene expression. (b) Two pairs of loxP-type recombination sequences (FLEX) for stable inversion proceeds in two steps: (1) inversion followed by (2) excision. loxP and lox2272 are orthogonal recombination sites. (Adapted from Kuhlman and Huang (111) and Atasoy et al. (110)).

currently intractable set of disorders, those in which photoreceptors degenerate within the retina $(129,130)$. To that end, the recent assessment of brain and immune function in nonhuman primates after ChR2 expression and activation, which showed a lack of harmful effects in a preliminary study (109), may pave the way toward new ideas for neural prosthetics for humans.

One strategy that has become widely popular, is to inject the brain of a mouse expressing Cre recombinase in a specific cell type, with a virus that either bears an opsin preceded by a lox-flanked stop cassette, or a virus that bears an opsin reversed and flanked by pairs of lox sites in a specific configuration (Fig. 7; $(110,111)$ ). Given the very large number of Cre transgenic mice in existence, and that are being generated, this strategy is likely to be very useful, at least for mice. Transgenic mice can be made that express Cre in extremely cell specific ways (e.g., through $3^{\prime}$ UTR knockins at the ends of cell typespecific genes), and then viruses can be rapidly made as new opsins are created, thus enabling cell type-specific expression without requiring the difficult process of trimming cell-specific promoters to fit within the small viral payload, or the difficult process of making transgenic mice for each new opsin tool that is developed (given the rapid pace of innovation, as shown in Sects. 1-4). 


\section{Hardware for Optical Neural Control}

For in vitro use, xenon lamps (e.g., Sutter DG-4, Till Photonics Polychrome) equipped with fast-moving mirrors or monochromators can be used for flexible delivery of fast (millisecondtimescale), brigh light pulses to biological samples on microscopes. Fluorescence filters can be used to deliver light of the appropriate wavelength (e.g., GFP excitation filter for ChR2, rhodamine or Texas Red excitation filter for Arch, Texas Red excitation filter for Halo, and GFP or YFP excitation filter for Mac). Recently, many companies such as Thorlabs have begun to sell LEDs or LED arrays compatible with microscope fluorescence illuminators, which sell for a small fraction of the price of a full lamp setup. Or fiber-coupled LEDs can simply be placed nearby to the sample (131). Confocal and two-photon microscopes, or more generally scanning laser methodologies, can be used to drive opsins, as have been described in a variety of papers $(112,132,133)$. Recently, digital micromirror displays (DMDs) have come forth as potentially useful for photostimulating in complex patterns, comprising millions of individual pixels that can be toggled either on or off $(134,135)$.

In vivo, the brain scatters light starting within a few hundred microns of an optical source, and absorbs light starting within a few millimeters of distance. Thus, most efforts for in vivo neuromodulation focus on delivering light to a volume of tissue, ranging from a very small volume containing a few hundred cells to a large volume (say, a few cubic millimeters) containing many thousands of cells. One widespread method is to use a laser coupled to an optical fiber (Fig. 8a shows a versatile setup that couples multiple colors of laser light into a single fiber; simpler commercially available single-color laser-coupled fibers with TTL control are also available), and to insert the fiber into a cannula implanted in the brain (Fig. 8 b shows a hand-built one for mice; commercial versions from companies such as Plastics One can also be built) or directly into the brain (Fig. 8c shows a setup for monkey), or to couple the fiber to an implanted fiber via a ferrule. An optical commutator (e.g., from Doric Lenses) can be placed between the fiber that inserts into the brain and the fiber that connects to the laser, to allow free rotation (66). Arrays of custom-targetable optical fibers, each coupled to a miniaturized light source (e.g., a raw die LED) and targeted to a unique target, will open up the ability to drive activity in complex 3D patterns, enabling the perturbation of complexly shaped structures as well as the ability to perturb targets in a patterned fashion $(136,137)$.

Aside from the key advantage of being able to manipulate a specific cell type, another key advantage of optical stimulation is the lack of electrical artifact as compared to conventional electrical 
a

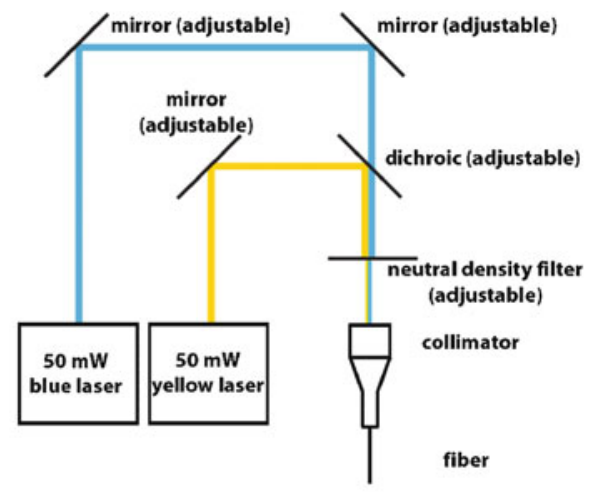

b

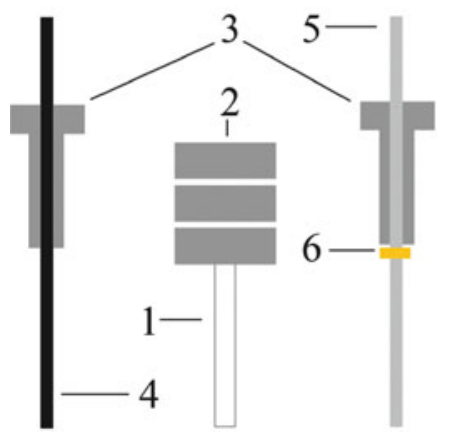

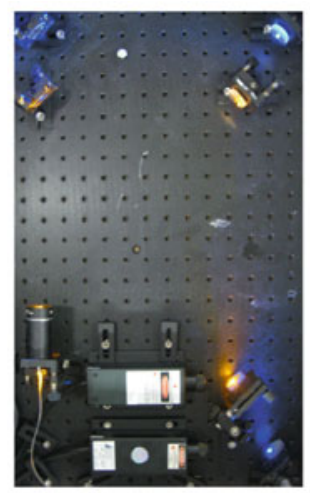

c

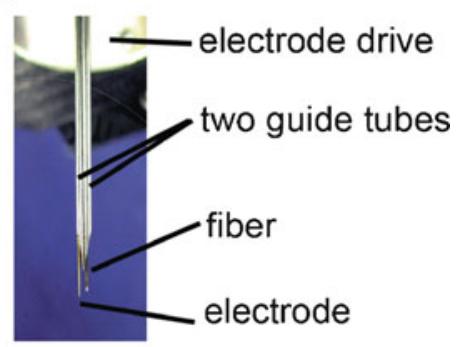

Fig. 8. (a) Schematic design (left) and picture (right) of an optics assembly used to couple blue and yellow laser light into a single optical fiber for in vivo neural modulation. A pictured assembly, lacking a neutral density filter, shows the hardware laid out on a standard optics breadboard. (b) Schematic design (left) and picture (right) of a system for targeting and securing optical fibers within the brain. A polyimide cannula $(1,250 \mu \mathrm{m}$ ID), designed to terminate at the locus of optical modulation, is epoxied to a stack of hex nuts $(2$, sized 2-56) which will be secured to the skull with dental cement. Vented screws (3, sized 2-56), which have holes in their centers, screw into the nuts while leaving a path open to the cannula. A dummy wire $(4,230 \mu \mathrm{m}$ stainless steel wire) may be epoxied to the screw to seal the craniotomy when the optics are not in use. An optical fiber ( $5,230 \mu \mathrm{m}$ OD silica fiber) is allowed free rotation without vertical displacement by a plastic washer ( 6 , homemade) which is epoxied to the fiber and sandwiched in between the vented screw above and the cannula below. (c) Apparatus for optical activation and electrical recording. Photograph, showing optical fiber (200 $\mu \mathrm{m}$ diameter) and electrode $(200 \mu \mathrm{m}$ shank diameter) in guide tubes. Adapted from Han et al. (19).

stimulation methods. However, despite the lack of electrical artifact, light does produce a voltage deflection when the electrode tip was illuminated (19, 138). For example, Fig. 9 shows traces recorded on a tungsten electrode in saline being illuminated by a pulsed laser beam, as adapted from (19). This voltage deflection slowly evolved over many tens of milliseconds, and accordingly was only recorded when the electrode voltage filtered at $0.7-170 \mathrm{~Hz}$ to examine local field potentials, (Fig. 9, top traces of each panel). This voltage deflection was not recorded when the electrode voltage was filtered at $250-8,000 \mathrm{~Hz}$ to detect spike signals; (Fig. 9, bottom traces of each panel). When light illuminated parts of the electrode other than the tip, no artifact was recorded; only illumination of the 
a

In saline

i

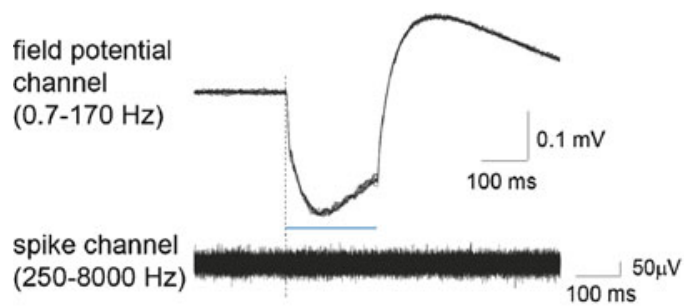

ii

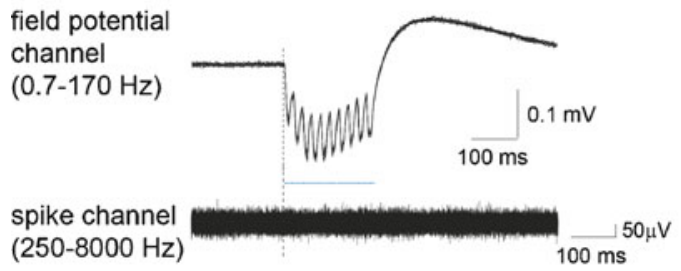

b

In brain

i

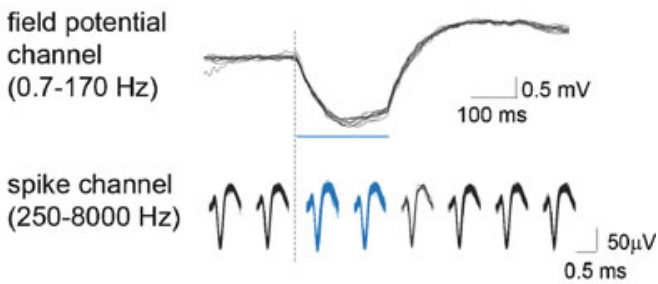

ii

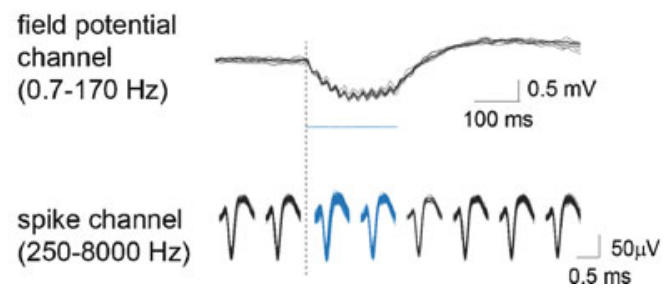

Fig. 9. Voltage deflections observed on tungsten electrodes immersed in saline (a) or brain (b), upon tip exposure to $200 \mathrm{~ms}$ blue light pulses (bi) or trains of $10 \mathrm{~ms}$ blue light pulses delivered at $50 \mathrm{~Hz}$ (bii). Light pulses are indicated by blue dashes. Electrode data was hardware filtered using two data acquisition channels operating in parallel, yielding a lowfrequency component ("field potential channel") and a high-frequency component ("spike channel"). For the "spike channel" traces taken in brain (b), spikes were grouped into $100 \mathrm{~ms}$ bins, and then the binned spikes were displayed beneath corresponding parts of the simultaneously acquired "field potential channel" signal (59 and 53 repeated light exposures for bi and bii respectively). (Shown are the spikes in eight such bins- the two bins before light onset, the two bins during the light delivery period, and the four bins after light cessation.) For all other signals shown, ten overlaid traces are plotted. From Han et al. (19).

tip-saline interface resulted in the voltage transient. This phenomenon is consistent with a classical photoelectrochemical finding, the Becquerel effect, in which illumination of an electrode placed in saline can produce a voltage on the electrode (139, 140). Consistent with the generality of the Becquerel effect as a property of electrode-electrolyte interfaces, this artifact is observed on various electrode materials, such as stainless steel, platinum-iridium, silver/ silver chloride, gold, nichrome, or copper. Similar slowly evolving voltage deflections were observed when tungsten electrodes were used to record neural activity in the brain, during optical stimulation. Because the optical artifact was slowly evolving over many tens of milliseconds, spike waveforms were detected without corruption by the artifact. However, local field potentials and field oscillations, which reflect coherent neural dynamics in the range of Hertz to tens of Hertz, may be difficult to isolate from this Becquerel artifact using the electrodes here tested. Notably, we have not seen the artifact with pulled glass micropipettes (such as previously used in (3) and (12), or in the mouse recordings with pulled glass pipettes in (109)). Thus, for recordings of local field potentials and other slow signals of importance for neuroscience, hollow glass electrodes may prove useful. 


\section{References}

1. Nagel G et al (2003) Channelrhodopsin-2, a directly light-gated cation-selective membrane channel. Proc Natl Acad Sci USA 100:13940-13945

2. Ishizuka T, Kakuda M, Araki R, Yawo H (2006) Kinetic evaluation of photosensitivity in genetically engineered neurons expressing green algae light-gated channels. Neurosci Res 54:85-94

3. Boyden ES, Zhang F, Bamberg E, Nagel G, Deisseroth K (2005) Millisecond-timescale, genetically targeted optical control of neural activity. Nat Neurosci 8:1263-1268

4. Li X et al (2005) Fast noninvasive activation and inhibition of neural and network activity by vertebrate rhodopsin and green algae channelrhodopsin. Proc Natl Acad Sci USA 102:17816-17821

5. Nagel G et al (2005) Light activation of channelrhodopsin-2 in excitable cells of Caenorhabditis elegans triggers rapid behavioral responses. Curr Biol 15:2279-2284

6. Wang $\mathrm{H}$ et al (2009) Molecular determinants differentiating photocurrent properties of two channelrhodopsins from chlamydomonas. J Biol Chem 284:5685-5696

7. Zhang F et al (2008) Red-shifted optogenetic excitation: a tool for fast neural control derived from Volvox carteri. Nat Neurosci 11:631-633

8. Berndt A, Yizhar O, Gunaydin LA, Hegemann P, Deisseroth K (2009) Bi-stable neural state switches. Nat Neurosci 12:229-234

9. Lewis TL Jr, Mao T, Svoboda K, Arnold DB (2009) Myosin-dependent targeting of transmembrane proteins to neuronal dendrites. Nat Neurosci 12:568-576

10. Lin JY, Lin MZ, Steinbach P, Tsien RY (2009) Characterization of engineered channelrhodopsin variants with improved properties and kinetics. Biophys J 96:1803-1814

11. Lanyi JK, Duschl A, Hatfield GW, May K, Oesterhelt D (1990) The primary structure of a halorhodopsin from Natronobacterium pharaonis. Structural, functional and evolutionary implications for bacterial rhodopsins and halorhodopsins. J Biol Chem 265:1253-1260

12. Han X, Boyden ES (2007) Multiple-color optical activation, silencing, and desynchronization of neural activity, with single-spike temporal resolution. PLoS One 2:e299

13. Zhang F et al (2007) Multimodal fast optical interrogation of neural circuitry. Nature 446:633-639
14. Bamberg E, Tittor J, Oesterhelt D (1993) Light-driven proton or chloride pumping by halorhodopsin. Proc Natl Acad Sci USA 90:639-643

15. Chow B et al (2010) High-performance genetically targetable optical neural silencing by light-driven proton pumps. Nature 463 (7277):98-102

16. Gradinaru V, Thompson KR, Deisseroth K (2008) eNpHR: a Natronomonas halorhodopsin enhanced for optogenetic applications. Brain Cell Biol 36:129-139

17. Zhao $S$ et al (2008) Improved expression of halorhodopsin for light-induced silencing of neuronal activity. Brain Cell Biol 36:141-154

18. Chow B, Han X, Qian X, Boyden ES (2009) High-performance halorhodopsin variants for improved genetically-targetable optical neural silencing. Frontiers in Systems Neuroscience. Conference Abstract: Computational and systems neuroscience. doi:10.3389/conf. neuro.10.2009.03.347

19. Han X, Qian X, Stern P, Chuong AS, Boyden ES (2009) Informational lesions: optical perturbation of spike timing and neural synchrony via microbial opsin gene fusions. Front Mol Neurosci. doi:10.3389/ neuro.02.012.2009

20. Henderson R, Schertler GF (1990) The structure of bacteriorhodopsin and its relevance to the visual opsins and other seven-helix G-protein coupled receptors. Philos Trans R Soc Lond B Biol Sci 326:379-389

21. Palczewski K (2006) G protein-coupled receptorrhodopsin. Annu Rev Biochem 75:743-767. doi:10.1146/annurev.biochem. 75.103004 .142743

22. Lanyi JK (2004) Bacteriorhodopsin. Annu Rev Physiol 66:665-688. doi:10.1146/ annurev.physiol.66.032102.150049

23. Lanyi JK (1986) Halorhodopsin: a light-driven chloride ion pump. Annu Rev Biophys Biophys Chem 15:11-28. doi:10.1146/annurev. bb.15.060186.000303

24. Essen LO (2002) Halorhodopsin: lightdriven ion pumping made simple? Curr Opin Struct Biol 12:516-522

25. Zemelman BV, Lee GA, Ng M, Miesenbock G (2002) Selective photostimulation of genetically chARGed neurons. Neuron 33:15-22

26. Lin B, Koizumi A, Tanaka N, Panda S, Masland RH (2008) Restoration of visual function in retinal degeneration mice by ectopic 
expression of melanopsin. Proc Natl Acad Sci USA 105:16009-16014

27. Kolbe M, Besir H, Essen LO, Oesterhelt D (2000) Structure of the light-driven chloride pump halorhodopsin at $1.8 \mathrm{~A}$ resolution. Science 288:1390-1396

28. Luecke H, Schobert B, Richter HT, Cartailler JP, Lanyi JK (1999) Structure of bacteriorhodopsin at $1.55 \mathrm{~A}$ resolution. J Mol Biol 291:899-911

29. Braiman MS, Stern LJ, Chao BH, Khorana HG (1987) Structure-function studies on bacteriorhodopsin. IV. Purification and renaturation of bacterio-opsin polypeptide expressed in Escherichia coli. J Biol Chem 262:9271-9276

30. Gilles-Gonzalez MA, Engelman DM, Khorana HG (1991) Structure-function studies of bacteriorhodopsin XV. Effects of deletions in loops $\mathrm{B}-\mathrm{C}$ and $\mathrm{E}-\mathrm{F}$ on bacteriorhodopsin chromophore and structure. J Biol Chem 266:8545-8550

31. Mogi T, Stern LJ, Chao BH, Khorana HG (1989) Structure-function studies on bacteriorhodopsin. VIII. Substitutions of the membrane-embedded prolines 50, 91, and 186: the effects are determined by the substituting amino acids. J Biol Chem 264:14192-14196

32. Mogi T, Marti T, Khorana HG (1989) Structure-function studies on bacteriorhodopsin. IX. Substitutions of tryptophan residues affect protein-retinal interactions in bacteriorhodopsin. J Biol Chem 264:14197-14201

33. Mogi T, Stern LJ, Hackett NR, Khorana HG (1987) Bacteriorhodopsin mutants containing single tyrosine to phenylalanine substitutions are all active in proton translocation. Proc Natl Acad Sci USA 84:5595-5599

34. Marti $\mathrm{T}$ et al (1991) Bacteriorhodopsin mutants containing single substitutions of serine or threonine residues are all active in proton translocation. J Biol Chem 266:6919-6927

35. Marinetti T, Subramaniam S, Mogi T, Marti T, Khorana HG (1989) Replacement of aspartic residues $85,96,115$, or 212 affects the quantum yield and kinetics of proton release and uptake by bacteriorhodopsin. Proc Natl Acad Sci USA 86:529-533

36. Mogi T, Stern LJ, Marti T, Chao BH, Khorana HG (1988) Aspartic acid substitutions affect proton translocation by bacteriorhodopsin. Proc Natl Acad Sci USA 85:4148-4152

37. Subramaniam S, Greenhalgh DA, Khorana HG (1992) Aspartic acid 85 in bacteriorho- dopsin functions both as proton acceptor and negative counterion to the Schiff base. J Biol Chem 267:25730-25733

38. Brown LS, Needleman R, Lanyi JK (1996) Interaction of proton and chloride transfer pathways in recombinant bacteriorhodopsin with chloride transport activity: implications for the chloride translocation mechanism. Biochemistry 35:16048-16054

39. Hegemann P, Oesterhelt D, Steiner M (1985) The photocycle of the chloride pump halorhodopsin. I: Azide-catalyzed deprotonation of the chromophore is a side reaction of photocycle intermediates inactivating the pump. EMBO J 4:2347-2350

40. Blanck A, Oesterhelt D (1987) The haloopsin gene. II. Sequence, primary structure of halorhodopsin and comparison with bacteriorhodopsin. EMBO J 6:265-273

41. Rudiger M, Oesterhelt D (1997) Specific arginine and threonine residues control anion binding and transport in the lightdriven chloride pump halorhodopsin. EMBO J 16:3813-3821

42. Varo G et al (1995) Light-driven chloride ion transport by halorhodopsin from Natronobacterium pharaonis. 1. The photochemical cycle. Biochemistry 34:14490-14499

43. Tittor J et al (1997) Chloride and proton transport in bacteriorhodopsin mutant D85T: different modes of ion translocation in a retinal protein. J Mol Biol 271:405-416

44. Tittor J, Oesterhelt D, Bamberg E (1995) Bacteriorhodopsin mutants D85N, D85T and $\mathrm{D} 85,96 \mathrm{~N}$ as proton pumps. Biophys Chem 56:153-157

45. Varo G, Needleman R, Lanyi JK (1995) Light-driven chloride ion transport by halorhodopsin from Natronobacterium pharaonis. 2. Chloride release and uptake, protein conformation change, and thermodynamics. Biochemistry 34:14500-14507

46. Berthold P et al (2008) Channelrhodopsin-1 initiates phototaxis and photophobic responses in Chlamydomonas by immediate light-induced depolarization. Plant Cell 20:1665-1677. doi:10.1105/tpc.108.057919

47. Sineshchekov OA, Govorunova EG, Spudich JL (2009) Photosensory functions of channelrhodopsins in native algal cells. Photochem Photobiol 85:556-563

48. Sineshchekov OA, Jung KH, Spudich JL (2002) Two rhodopsins mediate phototaxis to low- and high-intensity light in Chlamydomonas reinhardtii. Proc Natl Acad Sci USA 99:8689-8694 
49. Feldbauer K et al (2009) Channelrhodopsin2 is a leaky proton pump. Proc Natl Acad Sci USA 106:12317-12322

50. Nagel G et al (2002) Channelrhodopsin-1: a light-gated proton channel in green algae. Science 296:2395-2398

51. Ernst OP et al (2008) Photoactivation of channelrhodopsin. J Biol Chem 283:1637-1643

52. Zhang F, Wang LP, Boyden ES, Deisseroth K (2006) Channelrhodopsin-2 and optical control of excitable cells. Nat Methods 3:785-792

53. Huber D et al (2008) Sparse optical microstimulation in barrel cortex drives learned behaviour in freely moving mice. Nature 451:61-64

54. Bamann C, Kirsch T, Nagel G, Bamberg E (2008) Spectral characteristics of the photocycle of channelrhodopsin-2 and its implication for channel function. J Mol Biol 375:686-694

55. Nikolic K, Degenaar P, Toumazou C (2006) Modeling and engineering aspects of channelrhodopsin2 system for neural photostimulation. Conf Proc IEEE Eng Med Biol Soc 1:1626-1629

56. Tsunoda SP, Hegemann P (2009) Glu 87 of channelrhodopsin-1 causes pH-dependent color tuning and fast photocurrent inactivation. Photochem Photobiol 85:564-569

57. Ritter E, Stehfest K, Berndt A, Hegemann P, Bartl FJ (2008) Monitoring light-induced structural changes of channelrhodopsin- 2 by UV-visible and Fourier transform infrared spectroscopy. J Biol Chem 283:35033-35041. doi:10.1074/jbc.M806353200

58. Gunaydin LA et al (2010) Ultrafast optogenetic control. Nat Neurosci 13(3):387-92

59. Harwood JL, Guschina IA (2009) The versatility of algae and their lipid metabolism. Biochimie 91:679-684

60. Zipfel WR, Williams RM, Webb WW (2003) Nonlinear magic: multiphoton microscopy in the biosciences. Nat Biotechnol 21:1369-1377

61. Mohanty SK et al (2008) In-depth activation of channelrhodopsin 2-sensitized excitable cells with high spatial resolution using twophoton excitation with a near-infrared laser microbeam. Biophys J 95:3916-3926

62. Rickgauer JP, Tank DW (2009) Two-photon excitation of channelrhodopsin-2 at saturation. Proc Natl Acad Sci USA 106:15025-15030
63. Sineshchekov OA et al (2005) Rhodopsinmediated photoreception in cryptophyte flagellates. Biophys J 89:4310-4319

64. Sineshchekov OA, Litvin FF, Keszthelyi L (1990) Two components of photoreceptor potential in phototaxis of the flagellated green alga Haematococcus pluvialis. Biophys J 57:33-39

65. Litvin FF, Sineshchekov OA, Sineshchekov VA (1978) Photoreceptor electric potential in the phototaxis of the alga Haematococcus pluvialis. Nature 271:476-478

66. Gradinaru V et al (2007) Targeting and readout strategies for fast optical neural control in vitro and in vivo. $J$ Neurosci 27:14231-14238

67. Ihara K et al (1999) Evolution of the archaeal rhodopsins: evolution rate changes by gene duplication and functional differentiation. J Mol Biol 285:163-174

68. Mukohata Y, Ihara K, Tamura T, Sugiyama Y (1999) Halobacterial rhodopsins. J Biochem 125:649-657

69. Klare JP, Chizhov I, Engelhard M (2008) Microbial rhodopsins: scaffolds for ion pumps, channels, and sensors. Results Probl Cell Differ 45:73-122

70. Antón J et al (2005) Salinibacter ruber: genomics and biogeography. In: Gunde-Cimerman N, Plemenitas A, Oren A (eds) Adaptation to life in high salt concentrations in archaea, bacteria and eukarya. Kluwer Academic Publishers, Dordrecht, Netherlands, pp 257-266

71. Balashov SP et al (2005) Xanthorhodopsin: a proton pump with a light-harvesting carotenoid antenna. Science 309:2061-2064

72. Beja O, Spudich EN, Spudich JL, Leclerc M, DeLong EF (2001) Proteorhodopsin phototrophy in the ocean. Nature 411:786-789

73. Beja O et al (2000) Bacterial rhodopsin: evidence for a new type of phototrophy in the sea. Science 289:1902-1906

74. Friedrich T et al (2002) Proteorhodopsin is a light-driven proton pump with variable vectoriality. J Mol Biol 321:821-838

75. Kelemen BR, Du M, Jensen RB (2003) Proteorhodopsin in living color: diversity of spectral properties within living bacterial cells. Biochim Biophys Acta 1618:25-32

76. Kim SY, Waschuk SA, Brown LS, Jung KH (2008) Screening and characterization of proteorhodopsin color-tuning mutations 
in Escherichia coli with endogenous retinal synthesis. Biochim Biophys Acta 1777:504-513

77. Brown LS (2004) Fungal rhodopsins and opsin-related proteins: eukaryotic homologues of bacteriorhodopsin with unknown functions. Photochem Photobiol Sci 3:555-565

78. Waschuk SA, Bezerra AG, Shi L, Brown LS (2005) Leptosphaeria rhodopsin: bacteriorhodopsin-like proton pump from a eukaryote. Proc Natl Acad Sci USA 102:6879-6883. doi:10.1073/pnas.0409659102

79. Tsunoda SP et al (2006) $\mathrm{H}^{+}$-pumping rhodopsin from the marine alga Acetabularia. Biophys J 91:1471-1479

80. Yoshimura K, Kouyama T (2008) Structural role of bacterioruberin in the trimeric structure of archaerhodopsin-2. J Mol Biol 375:1267-1281

81. Enami N et al (2006) Crystal structures of archaerhodopsin-1 and -2: common structural motif in archaeal light-driven proton pumps. J Mol Biol 358:675-685

82. Seki A et al (2007) Heterologous expression of Pharaonis halorhodopsin in Xenopus laevis oocytes and electrophysiological characterization of its light-driven $\mathrm{Cl}^{-}$pump activity. Biophys J 92:2559-2569

83. Okuno D, Asaumi M, Muneyuki E (1999) Chloride concentration dependency of the electrogenic activity of halorhodopsin. Biochemistry 38:5422-5429

84. Muneyuki E, Shibazaki C, Wada Y, Yakushizin M, Ohtani H (2002) $\mathrm{Cl}(-)$ concentration dependence of photovoltage generation by halorhodopsin from Halobacterium salinarum. Biophys J 83:1749-1759

85. Gradinaru Vet al (2010) Molecular and cellular approaches for diversifying and extending optogenetics. Cell 141(1):154-65

86. Baliga NS et al (2004) Genome sequence of Haloarcula marismortui: a halophilic archaeon from the Dead sea. Genome Res 14:2221-2234

87. Tonnesen J, Sorensen AT, Deisseroth K, Lundberg C, Kokaia M (2009) Optogenetic control of epileptiform activity. Proc Natl Acad Sci USA 106:12162-12167

88. Bernstein JG et al (2008) Prosthetic systems for therapeutic optical activation and silencing of genetically-targeted neurons. Proc Soc Photo Opt Instrum Eng 6854:68540H

89. Ludmann K, Ibron G, Lanyi JK, Varo G (2000) Charge motions during the photocycle of pharaonis halorhodopsin. Biophys J 78:959-966
90. Chizhov I, Engelhard M (2001) Temperature and halide dependence of the photocycle of halorhodopsin from Natronobacterium pharaonis. Biophys J 81:1600-1612

91. Ming M et al (2006) pH dependence of lightdriven proton pumping by an archaerhodopsin from Tibet: comparison with bacteriorhodopsin. Biophys J 90:3322-3332

92. Lukashev EP et al (1994) pH dependence of the absorption spectra and photochemical transformations of the archaerhodopsins. Photochem Photobiol 60:69-75

93. Lanyi JK (2006) Proton transfers in the bacteriorhodopsin photocycle. Biochimica et Biophysica Acta (BBA) - Bioenergetics 1757:1012-1018

94. Bevensee MO, Cummins TR, Haddad GG, Boron WF, Boyarsky G (1996) pH regulation in single CAl neurons acutely isolated from the hippocampi of immature and mature rats. J Physiol 494(Pt 2):315-328

95. Chesler M (2003) Regulation and modulation of $\mathrm{pH}$ in the brain. Physiol Rev 83:1183-1221

96. Meyer TM, Munsch T, Pape HC (2000) Activity-related changes in intracellular $\mathrm{pH}$ in rat thalamic relay neurons. Neuroreport 11:33-37

97. Trapp S, Luckermann M, Brooks PA, Ballanyi K (1996) Acidosis of rat dorsal vagal neurons in situ during spontaneous and evoked activity. J Physiol 496(Pt 3):695-710

98. Brown LS et al (1995) Glutamic acid 204 is the terminal proton release group at the extracellular surface of bacteriorhodopsin. J Biol Chem 270:27122-27126

99. Phatak P, Ghosh N, Yu H, Cui Q, Elstner M (2008) Amino acids with an intermolecular proton bond as proton storage site in bacteriorhodopsin. Proc Natl Acad Sci USA 105:19672-19677

100. Henderson R et al (1990) Model for the structure of bacteriorhodopsin based on high-resolution electron cryo-microscopy. J Mol Biol 213:899-929

101. Man-Aharonovich D et al (2004) Characterization of RS29, a blue-green proteorhodopsin variant from the Red Sea. Photochem Photobiol Sci 3:459-462

102. Sasaki J et al (1995) Conversion of bacteriorhodopsin into a chloride ion pump. Science 269:73-75

103. Iwamoto $M$ et al (2004) Proton release and uptake of pharaonis phoborhodopsin (sensory rhodopsin II) reconstituted into phospholipids. Biochemistry 43:3195-3203 
104. Sudo Y, Iwamoto M, Shimono K, Sumi M, Kamo N (2001) Photo-induced proton transport of pharaonis phoborhodopsin (sensory rhodopsin II) is ceased by association with the transducer. Biophys J 80:916-922

105. Boichenko VA, Wang JM, Antón J, Lanyi JK, Balashov SP (2006) Functions of carotenoids in xanthorhodopsin and archaerhodopsin, from action spectra of photoinhibition of cell respiration. Biochimica et Biophysica Acta (BBA) - $\quad$ Bioenergetics 1757:1649-1656

106. Serrano EE, Zeiger E, Hagiwara S (1988) Red light stimulates an electrogenic proton pump in Vicia guard cell protoplasts. Proc Natl Acad Sci USA 85:436-440

107. Moreau CJ, Dupuis JP, Revilloud J, Arumugam K, Vivaudou M (2008) Coupling ion channels to receptors for biomolecule sensing. Nat Nanotechnol 3:620-625

108. Wang $\mathrm{H}$ et al (2007) High-speed mapping of synaptic connectivity using photostimulation in channelrhodopsin-2 transgenic mice. Proc Natl Acad Sci USA 104:8143-8148

109. Han X et al (2009) Millisecond-timescale optical control of neural dynamics in the nonhuman primate brain. Neuron 62:191-198

110. Atasoy D, Aponte Y, Su HH, Sternson SM (2008) A FLEX switch targets channelrhodopsin-2 to multiple cell types for imaging and long-range circuit mapping. J Neurosci 28:7025-7030

111. Kuhlman SJ, Huang ZJ (2008) High-resolution labeling and functional manipulation of specific neuron types in mouse brain by Creactivated viral gene expression. PLoS One 3: e2005

112. Petreanu L, Huber D, Sobczyk A, Svoboda K (2007) Channelrhodopsin-2-assisted circuit mapping of long-range callosal projections. Nat Neurosci 10:663-668

113. Schroll C et al (2006) Light-induced activation of distinct modulatory neurons triggers appetitive or aversive learning in Drosophila larvae. Curr Biol 16:1741-1747

114. Douglass AD, Kraves S, Deisseroth K, Schier AF, Engert F (2008) Escape behavior elicited by single, channelrhodopsin-2-evoked spikes in zebrafish somatosensory neurons. Curr Biol 18:1133-1137

115. Yan W et al (2001) Cloning and characterization of a human beta, beta-carotene-15,15' dioxygenase that is highly expressed in the retinal pigment epithelium. Genomics 72:193-202

116. Dittgen T et al (2004) Lentivirus-based genetic manipulations of cortical neurons and their optical and electrophysiological monitoring in vivo. Proc Natl Acad Sci USA 101:18206-18211

117. Chhatwal JP, Hammack SE, Jasnow AM, Rainnie DG, Ressler KJ (2007) Identification of cell-type-specific promoters within the brain using lentiviral vectors. Gene Ther 14:575-583

118. Adamantidis AR, Zhang F, Aravanis AM, Deisseroth K, de Lecea L (2007) Neural substrates of awakening probed with optogenetic control of hypocretin neurons. Nature 450:420-424

119. Tan W et al (2008) Silencing preBotzinger complex somatostatin-expressing neurons induces persistent apnea in awake rat. Nat Neurosci 1 1:538-540

120. Nathanson JL, Yanagawa Y, Obata K, Callaway EM (2009) Preferential labeling of inhibitory and excitatory cortical neurons by endogenous tropism of adeno-associated virus and lentivirus vectors. Neuroscience $161: 441-450$

121. Wickersham IR, Finke S, Conzelmann KK, Callaway EM (2007) Retrograde neuronal tracing with a deletion-mutant rabies virus. Nat Methods 4:47-49

122. Stachler MD, Chen I, Ting AY, Bartlett JS (2008) Site-specific modification of AAV vector particles with biophysical probes and targeting ligands using biotin ligase. Mol Ther 16:1467-1473

123. Toni $\mathrm{N}$ et al (2008) Neurons born in the adult dentate gyrus form functional synapses with target cells. Nat Neurosci 11:901-907

124. Wickersham IR et al (2007) Monosynaptic restriction of transsynaptic tracing from single, genetically targeted neurons. Neuron 53:639-647

125. Banfield BW, Kaufman JD, Randall JA, Pickard GE (2003) Development of pseudorabies virus strains expressing red fluorescent proteins: new tools for multisynaptic labeling applications. J Virol 77:10106-10112

126. Foust KD et al (2009) Intravascular AAV9 preferentially targets neonatal neurons and adult astrocytes. Nat Biotechnol 27:59-65

127. Chan SC, Bernstein JG, Boyden ES (2010) Scalable fluidic injector arrays for viral targeting of intact 3-D brain circuits. J Vis Exp 35:1489

128. (2007) Retracing events. Nat Biotechnol 25:949

129. Bi A et al (2006) Ectopic expression of a microbial-type rhodopsin restores visual responses in mice with photoreceptor degeneration. Neuron 50:23-33 
130. Lagali PS et al (2008) Light-activated channels targeted to $\mathrm{ON}$ bipolar cells restore visual function in retinal degeneration. Nat Neurosci 11:667-675

131. Campagnola L, Wang H, Zylka MJ (2008) Fiber-coupled light-emitting diode for localized photostimulation of neurons expressing channelrhodopsin-2. J Neurosci Methods 169:27-33

132. Rickgauer JP, Tank DW. In: Neuroscience 2008. Society for Neuroscience

133. Petreanu L, Mao T, Sternson SM, Svoboda K (2009) The subcellular organization of neocortical excitatory connections. Nature 457:1142-1145

134. Farah N, Reutsky I, Shoham S (2007) Patterned optical activation of retinal ganglion cells. Conf Proc IEEE Eng Med Biol Soc 2007:6369-6371

135. Guo ZV, Hart AC, Ramanathan S (2009) Optical interrogation of neural circuits in Caenorbabditis elegans. Nat Methods 6:891-896

136. Bernstein J et al (2008) A scalable toolbox for systematic, cell-specific optical control of entire 3-D neural circuits in the intact mammalian brain. Society for Neuroscience

137. Bernstein JG et al (2009) Modulation of fear behavior via optical fiber arrays targeted to bilateral prefrontal cortex. Society for Neuroscience

138. Ayling OG, Harrison TC, Boyd JD, Goroshkov A, Murphy TH (2009) Automated lightbased mapping of motor cortex by photoactivation of channelrhodopsin-2 transgenic mice. Nat Methods 6:219-224

139. Honda K (2004) Dawn of the evolution of photoelectrochemistry. J Photochem Photobiol A Chem 166:63-68

140. Gratzel M (2001) Photoelectrochemical cells. Nature 414:338-344

141. Chow YC, Han X, Bernstein JG, Monahan PE, Boyden ES (2011) Light-activated ion pumps and channels for temporally precise optical control of activity in genetically targeted neurons. In: Chambers JJ, Kramer RH (eds) Photosensitive molecules for controlling biological function. Neuromethods, vol 55. Springer, New York, doi: 10.1007/978-1-61779-031-7 